\title{
Intersections of Lagrangian submanifolds and the Mel'nikov 1-form
}

\author{
Nicolas Roy ${ }^{* \dagger}$
}

8th April 2018

\begin{abstract}
We make explicit the geometric content of Mel'nikov's method for detecting heteroclinic points between transversally hyperbolic periodic orbits. After developing the general theory of intersections for pairs of families of Lagrangian submanifolds $\mathcal{N}_{\varepsilon}^{ \pm}$, with $\mathcal{N}_{0}^{+}=\mathcal{N}_{0}^{-}$and constrained to live in an auxiliary family of submanifolds, we explain how the heteroclinic orbits of a given Hamiltonian system are detected by the zeros of the Mel'nikov 1-form. This 1-form admits an integral expression which is non-convergent in general. We discuss different solutions to this convergence problem.
\end{abstract}

\section{Introduction}

In his article [11], Mel'nikov introduced a method for studying time-periodic perturbations $H_{\varepsilon}(x, \xi, t)=H_{0}(x, \xi)+\varepsilon H_{1}(x, \xi, t)$ of 2-dimensional time-independent Hamiltonian systems. The author considers the case where $H_{0}$ has a hyperbolic fixed point $m_{0} \in \mathbb{R}^{2}$ such that (one "half" of) its stable manifold coincides with (one "half" of) its unstable manifold, as depicted on the picture below.

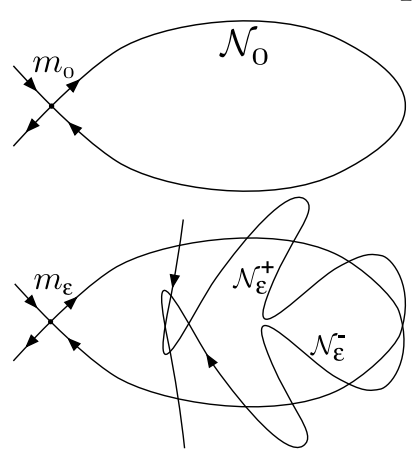

Let us denote this manifold by $\mathcal{N}_{0}$. For studying the timedependent perturbations of $H_{0}$, one might consider a section at time $t=0$ of the system in $\mathbb{R}^{2} \times S^{1}$, given by the time 1 flow $\phi_{X_{H_{\varepsilon}}}^{t=1}$. Because of the structural stability of hyperbolic points, there is a smooth family $m_{\varepsilon}$ of hyperbolic points for the map $\phi_{X_{H_{\varepsilon}}}^{1}$. Furthermore, the hyperbolicity implies the existence of a smooth family of stable (resp. unstable) manifolds $\mathcal{N}_{\varepsilon}^{+}$(resp. $\mathcal{N}_{\varepsilon}^{-}$) for $m_{\varepsilon}$. However, as soon as $\varepsilon \neq 0$, they might not coincide and their intersections (called homoclinic points) form in general a very complicated set. See the pic-

ture on the left.

\footnotetext{
${ }^{*}$ We are very grateful to Y. Colin de Verdière who pointed this issue out to us.

${ }^{\dagger}$ We would like to thank the referee for his careful reading of the manuscript and for pointing out several inaccuracies and missing bibliographic references.
} 
This phenomenon, referred to as the "homoclinic entanglement", is the sign of the chaotic behaviour of the system near $m_{0}$. It is also known to be the key feature of Arnold's diffusion (see e.g. [3]). In order to detect the positions of the homoclinic points of $m_{\varepsilon}$, Mel'nikov defined the function

$$
M(t)=\int_{-\infty}^{+\infty}\left\{H_{1}(t+s), H_{0}\right\}(m(s)) d s,
$$

where $m(s)$ is the trajectory on $\mathcal{N}_{0}$ under the unperturbed dynamics of $H_{0}$ starting from a chosen point $m \in \mathcal{N}_{0}$. This point plays the role of an origin on $\mathcal{N}_{0}$ and $t$ is a coordinate. Mel'nikov shows that the non-degenerate zeros of $M$ describe at first order in $\varepsilon$ the position of the homoclinic points of the perturbed hyperbolic point $m_{\varepsilon}$. The main feature of the expression of $M$ is that the only flow that one has to integrate is the one of $H_{0}$, i.e.,, the unperturbed dynamics, which is supposed to be well understood. On the other hand, one knows that such a time periodic perturbed system can be rewritten as an autonomous one, thru a standard procedure. Namely, one takes the product of the initial symplectic manifold (here simply $\mathbb{R}^{2}$ ) with $T^{*} S^{1}$, where the $S^{1}$ factor corresponds to the $t$ variable. In the extended system, the hyperbolic fixed point $m_{\varepsilon}$ becomes a transversally hyperbolic periodic orbit $\gamma_{\varepsilon}$, whose stable and unstable manifolds intersect along trajectories homoclinic to $\gamma_{\varepsilon}$.

The main goal of this article is to clarify the geometric content of Mel'nikov's method, which extends to higher-dimensional systems on general symplectic manifolds, for detecting heteroclinic (and not only homoclinic) orbits linking two periodic orbits. The Mel'nikov's method has actually two separate aspects. First, the heteroclinic orbits are in correspondence with the zeros of a geometric object, namely the Mel'nikov's 1-form. Second, one tries to give this 1-form an integral expression involving only the flow of the unperturbed dynamics. These two issues roughly correspond to the main two sections of this paper.

The extension of Mel'nikov's technique for detecting heteroclinic orbits linking two transversally hyperbolic periodic orbits or tori, rather than hyperbolic points, has been considered by many authors, e.g. [2, 5, 6, , 7, 9, 10, 14]. But, they all consider dynamical systems with the common feature that there is an explicit separation between the "longitudinal" and "transversal" variables, corresponding respectively to the motion along the tori (or the periodic orbits) and the hyperbolic transversal motion. It turns out that resorting to explicit coordinates has several drawbacks we would like to point out now.

- First of all, this assumption is unnecessary and actually goes against a satisfactory understanding of the geometry underlying this method. One aim of this paper is to describe the geometric objects involved in Mel'nikov's method without reference to any coordinate system. In particular, as a multidimensional generalisation of the Mel'nikov function, the authors introduce a "Mel'nikov vector", whereas the correct geometric object is rather a 1-form, as we explain throughout this paper. The use of a 1-form is in fact very natural since Mel'nikov's method deals with deformations of Lagrangian submanifolds (the stable and unstable ones) and 
it is well-known [13] that the deformation theory of Lagrangian submanifolds is parameterised by closed 1-forms. The Mel'nikov's 1-form is thus closed and it is actually exact for geometrical reasons explained in Section 2.2.3. We believe that this clarifies the statement "The Mel'nikov vector is a gradient" which, in the literature, seems to be true for a bit obscure reasons. In fact, this is always true and not only in the particular models people studied.

- Second, these particular models (with a separation between the longitudinal and transversal motions) dismiss a large class of systems. Indeed, it is well-known from different studies of completely integrable systems [4, 12] that the local model near a transversally hyperbolic invariant $m$-dimensional torus is not always $\mathbb{T}^{m} \times$ $\mathbb{R}^{m} \times \mathbb{R}^{2 n}$ (as in the mentioned particular models) but may be a quotient of that by a finite group. For example, in dimension 4 , it happens that the local stable and unstable manifolds of a periodic orbit is not diffeomorphic to the cylinder but rather to the Möbius strip ${ }^{1}$.

- Third, these particular systems are highly non-generic in the heteroclinic case. Indeed, they have the feature that the heteroclinic manifolds link two tori with the same Diophantine property. For example, in the case of periodic orbits (instead of tori), this means that the orbits have the same period. Generically, the periods are different and this prevents us from expressing the Mel'nikov 1-form in terms of an integral over the unperturbed flow. This issue is treated in Section 2.3.4.

The general tool we will rely on is the intersection theory for pairs $\left(\mathcal{N}_{\varepsilon}^{+}, \mathcal{N}_{\varepsilon}^{-}\right)$of Lagrangian submanifolds which coincide for $\varepsilon=0$ and which are constrained to live in an auxiliary submanifold $\mathcal{N}_{\varepsilon}^{ \pm} \subset \mathcal{P}_{\varepsilon}$ for all $\varepsilon$. Indeed, stable and unstable manifolds of transversally hyperbolic periodic orbits are Lagrangian and confined at least in an energy level $\left\{H_{\varepsilon}=c s t\right\}$. For this particular intersection theory, one has to introduce a suitable "transversality" condition at $\varepsilon=0$ (roughly speaking, a condition on the variations " $\frac{d \mathcal{N}_{\varepsilon}^{ \pm}}{d \varepsilon}$ ") in order to insure transversality of $\mathcal{N}_{\varepsilon}^{+}$and $\mathcal{N}_{\varepsilon}^{-}$in $\mathcal{P}_{\varepsilon}$ for $\varepsilon \neq 0$, since the usual transversality hypothesis is obviously not fulfilled at $\varepsilon=0$. This theory, which actually applies to any pair of Lagrangian submanifolds regardless to their stable/unstable feature, is developed in Section 11. It is shown that investigating the intersections of $\mathcal{N}_{\varepsilon}^{+}$and $\mathcal{N}_{\varepsilon}^{-}$for $\varepsilon \neq 0$ amounts to looking for the "non-degenerate" zeros of a 1-form $\beta$ defined on $\mathcal{N}_{0}^{+}=\mathcal{N}_{0}^{-}$, which we call the Mel'nikov 1-form despite this name takes on its full meaning only when $\mathcal{N}_{\varepsilon}^{+}$and $\mathcal{N}_{\varepsilon}^{-}$are the stable and unstable manifolds of two transversally hyperbolic periodic orbits $\gamma_{\varepsilon}^{ \pm}$. In that case, the intersections of $\mathcal{N}_{\varepsilon}^{+}$and $\mathcal{N}_{\varepsilon}^{-}$are thus heteroclinic points between $\gamma_{\varepsilon}^{+}$and $\gamma_{\varepsilon}^{-}$, or homoclinic points in case there is only one periodic orbit $\gamma_{\varepsilon}^{+}=\gamma_{\varepsilon}^{-}$.

This is the topic of Section 2, where we apply the theory developed in Section 1 to this heteroclinic/homoclinic situation. We will focus on the following questions.

\footnotetext{
${ }^{1}$ See [4] for a precise description of such systems. See also the end of section 2.1.1] for a picture of this situation.
} 
When the unperturbed Hamiltonian $H_{0}$ is completely integrable (this is automatic for 2-dimensional systems), i.e., admits a momentum map $\mathbf{A}=\left(A_{1}, \ldots, A_{d}\right)$, then one can compute the Mel'nikov 1-form $\beta$ thru the evaluations $\beta\left(X_{A_{j}}\right)$. This shows in particular that in the near-integrable case, the splitting of the stable and unstable manifolds is completely described by the integrals of motion of the unperturbed Hamiltonian $H_{0}$. Beside this, it turns out that the functions $\beta\left(X_{A_{j}}\right)$ have an integral expression involving only the flow of $H_{0}$. Unfortunately, these integrals do not converge in general. Then, we discuss what are the different solutions to this convergence problem, namely either assuming that the perturbation $H_{\varepsilon}-H_{0}$ is critical on both orbits $\gamma_{0}^{ \pm}$or choosing the $A_{j}$ 's critical on $\gamma_{0}^{ \pm}$. The latter works perfectly in the homoclinic situation, but we explain that in the heteroclinic one, there is usually not enough independent such $A_{j}$ 's to determine the Mel'nikov 1-form. We show however that there is a special case (to which belong the time-periodic systems) for which there is precisely enough $A_{j}$ 's critical on $\gamma_{0}^{ \pm}$to compute $\beta$. This question is usually ignored in the literature since the authors consider either the homoclinic situation or periodically forced systems.

\section{Intersections of families of Lagrangian submanifolds}

We forget for the moment the heteroclinic theory of transversally hyperbolic orbits and we begin with the intersection theory for some families of compact submanifolds $\mathcal{N}_{\varepsilon}^{ \pm}$ in a given manifold $\mathcal{M}$. All the manifolds under consideration are smooth. As well, we assume that the families depend smoothly on the deformation parameter $\varepsilon$, in the sense that the union $\bigcup_{\varepsilon}\left(\mathcal{N}_{\varepsilon}^{ \pm} \times\{\varepsilon\}\right)$ is a smooth submanifold of $\mathcal{M} \times \mathbb{R}$. From now on, both these smoothness conditions will always be implicitly assumed.

It is well-known that whenever $\mathcal{N}_{0}^{+}$and $\mathcal{N}_{0}^{-}$intersect transversally at some point $m$, i.e., $T_{m} \mathcal{N}_{0}^{+} \oplus T_{m} \mathcal{N}_{0}^{-}=T_{m} \mathcal{M}$, then in a neighbourhood of $m$, the intersection $\mathcal{N}_{0}^{+} \cap \mathcal{N}_{0}^{-}$ is a smooth submanifold of dimension equal to $\operatorname{dim} \mathcal{N}_{0}^{+}+\operatorname{dim} \mathcal{N}_{0}^{-}-\operatorname{dim} \mathcal{M}$. Moreover, $\mathcal{N}_{\varepsilon}^{+} \cap \mathcal{N}_{\varepsilon}^{-}$is a smooth family of submanifolds of $\mathcal{M}$, for small enough $\varepsilon$.

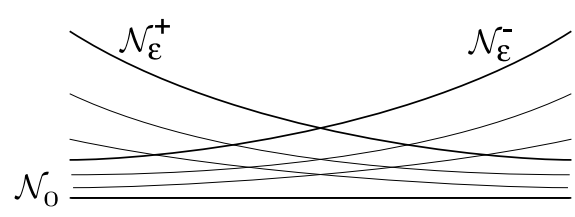

As mentioned in the introduction, we need to consider the situation where $\mathcal{N}_{\varepsilon}^{+}$and $\mathcal{N}_{\varepsilon}^{-}$are deformations of the same $\mathcal{N}_{0}$. Such families are obviously not transverse for $\varepsilon=0$, but a suitable transversality condition on the "first derivatives $\frac{d}{d \varepsilon} \mathcal{N}_{\varepsilon}^{ \pm}$" can be introduced to describe the intersection $\mathcal{N}_{\varepsilon}^{+} \cap \mathcal{N}_{\varepsilon}^{-}$for $\varepsilon \neq 0$. This issue is addressed in Section 1.1.

On the other hand, if we know a priori that $\mathcal{N}_{\varepsilon}^{+}$and $\mathcal{N}_{\varepsilon}^{-}$are constrained to live in an intermediate submanifold $\mathcal{P}_{\varepsilon}$, the smoothness of the intersection $\mathcal{N}_{\varepsilon}^{+} \cap \mathcal{N}_{\varepsilon}^{-}$can be insured by a "infinitesimal transversality" condition in $\mathcal{P}_{0}$. This is precisely the case for the Mel'nikov situation where the families under consideration are included in a level set of the Hamiltonian function $H_{\varepsilon}$. This question is considered in Section 1.2 .

Eventually, in the symplectic framework, the intersection theory for Lagrangian submanifolds is somehow simpler and it is well-described by the Mel'nikov 1-form, a dif- 
ferential form on $\mathcal{N}_{0}$, as we discuss in Section 1.3

\subsection{Infinitesimally transverse intersections}

\subsubsection{Generating flows for families of submanifolds}

First, we need to parameterise the families of submanifolds with families of diffeomorphisms in the following way.

Definition 1. Let $\mathcal{N}_{\varepsilon} \subset \mathcal{M}$ be a family of compact submanifolds. A (time-dependent) vector field $X_{\varepsilon}$ is said to generate $\mathcal{N}_{\varepsilon}$ if its flow $\phi_{X_{\varepsilon}}^{\varepsilon}$ satisfies $\phi_{X_{\varepsilon}}^{\varepsilon}\left(\mathcal{N}_{0}\right)=\mathcal{N}_{\varepsilon}$ and if $X_{0}$ is not tangent to $\mathcal{N}_{0}$ whenever it is non-zero, i.e.,"

$$
X_{0}(m) \in T_{m} \mathcal{N}_{0} \Longrightarrow X_{0}(m)=0 .
$$

We will also say that the flow $\phi_{X_{\varepsilon}}^{\varepsilon}$ generates $\mathcal{N}_{\varepsilon}$.

Notice that in general it is impossible to a choose a time-independent vector field to generate a given family $\mathcal{N}_{\varepsilon}$, whereas there always exists a time-dependent one, as the next lemma shows.

Lemma 2. Let $\mathcal{N}_{\varepsilon} \subset \mathcal{M}$ be a family of compact submanifolds. Then there exists a vector field $X_{\varepsilon}$ generating $\mathcal{N}_{\varepsilon}$. Moreover, when one is given two deformations $\mathcal{N}_{\varepsilon}^{ \pm}$of the same $\mathcal{N}_{0}:=\mathcal{N}_{0}^{+}=$ $\mathcal{N}_{0}^{-}$, then there exist generating vector fields $X_{\varepsilon}^{ \pm}$such that $X_{0}^{+}-X_{0}^{-}$is not tangent to $\mathcal{N}_{0}$ whenever it is non-zero.

Proof. The Tubular Neighbourhood Theorem says that there is a neighborhood $\mathcal{O} \subset \mathcal{M}$ of $\mathcal{N}_{0}$, a vector bundle $E$ over $\mathcal{N}_{0}$, a neighborhood $\tilde{\mathcal{O}} \subset E$ of the zero-section and a diffeomorphism $\chi: \mathcal{O} \rightarrow \tilde{\mathcal{O}}$ which sends $\mathcal{N}_{0}$ to the zero-section. One can assume $\mathcal{O}$ and $\tilde{\mathcal{O}}$ are compact. For small enough $\varepsilon$, the submanifolds $\mathcal{N}_{\varepsilon}^{ \pm}$lie in $\mathcal{O}$. Thru the map $\chi$, one obtains families of submanifolds $\tilde{\mathcal{N}}_{\varepsilon}^{ \pm}=\chi\left(\mathcal{N}_{\varepsilon}^{ \pm}\right) \subset \tilde{\mathcal{O}}$ close to the zero-section, hence they are graphs of sections of $E$. Therefore, there exist particular vector fields $\tilde{Y}_{\varepsilon}^{ \pm}$ generating $\tilde{\mathcal{N}}_{\varepsilon}^{ \pm}$, namely those associated to vertical translations. These vector fields are vertical and thus not tangent to $\mathcal{N}_{0}$ whenever they do not vanish. The same is true for the difference $Y_{\varepsilon}^{+}-Y_{\varepsilon}^{-}$. Now, define $\tilde{X}_{\varepsilon}^{ \pm}=f \tilde{Y}_{\varepsilon}^{ \pm}$, with $f \in C_{0}^{\infty}(E)$ a smooth function with support in $\tilde{\mathcal{O}}$ and equal to 1 in a (smaller) neighborhood of the zero section. By construction, for small enough $\varepsilon$, the vector fields $X_{\varepsilon}^{ \pm}=\chi_{*}^{-1}\left(\tilde{X}_{\varepsilon}^{ \pm}\right)$on $\mathcal{M}$ generate $\mathcal{N}_{\varepsilon}^{ \pm}$ in the sense of Definition 1

In all the following, we will always choose implicitly generating vector fields with the property of Lemma 2 For a given family $\mathcal{N}_{\varepsilon}$, the choice of a generating vector field is of course not unique, but different choices are related as follows.

Lemma 3. Let $\mathcal{N}_{\varepsilon} \subset \mathcal{M}$ be a family of compact submanifolds. If two vector fields $X_{\varepsilon}$ and $Y_{\varepsilon}$ generate $\mathcal{N}_{\varepsilon}$, then the difference $X_{\varepsilon}-Y_{\varepsilon}$ is tangent to $\mathcal{N}_{\varepsilon}$ for all $\varepsilon$. 
Proof. Denote by $\phi^{\varepsilon}\left(\right.$ resp. $\left.\varphi^{\varepsilon}\right)$ the flow of $X_{\varepsilon}$ (resp. $Y_{\varepsilon}$ ). The vector field of the flow $\left(\phi^{\varepsilon}\right)^{-1}$ is equal to $-\left(\phi^{\varepsilon}\right)_{*}^{-1}\left(X_{\varepsilon}\right)$ and therefore, the composition $\psi^{\varepsilon}=\left(\phi^{\varepsilon}\right)^{-1} \circ \varphi^{\varepsilon}$ is the flow of the vector field $\left(\phi^{\varepsilon}\right)_{*}^{-1}\left(-X_{\varepsilon}+Y_{\varepsilon}\right)$. On the other hand, $\psi^{\varepsilon}$ obviously sends $\mathcal{N}_{0}$ to itself and its vector field is thus tangent to $\mathcal{N}_{0}$. This implies that $X_{\varepsilon}-Y_{\varepsilon}$ is tangent to $\phi^{\varepsilon}\left(\mathcal{N}_{0}\right)=\mathcal{N}_{\varepsilon}$, for all $\varepsilon$.

\subsubsection{Infinitesimal transversality}

From now on, we consider two families $\mathcal{N}_{\varepsilon}^{+}$and $\mathcal{N}_{\varepsilon}^{-}$which are deformations of the same submanifold $\mathcal{N}_{0}:=\mathcal{N}_{0}^{+}=\mathcal{N}_{0}^{-}$. Let us now introduce the suitable transversality condition to describe the intersections of $\mathcal{N}_{\varepsilon}^{+}$and $\mathcal{N}_{\varepsilon}^{-}$.

Definition 4. Let $\mathcal{N}_{\varepsilon}^{ \pm} \subset \mathcal{M}$ be two families of compact submanifolds generated by vector fields $X_{\varepsilon}^{ \pm}$. We say that a point $m \in \mathcal{N}_{0}$ is an infinitesimal intersection of $\mathcal{N}_{\varepsilon}^{+}$and $\mathcal{N}_{\varepsilon}^{-}$if $X_{0}^{+}=X_{0}^{-}$at $m$.

Lemma 5. The notion of "infinitesimal intersection" is well-defined, i.e., independent of the choice of $X_{\varepsilon}^{ \pm}$.

Proof. Let $X_{\varepsilon}^{+}$(resp. $X_{\varepsilon}^{-}$) be a vector field generating $\mathcal{N}_{\varepsilon}^{+}$(resp. $\mathcal{N}_{\varepsilon}^{-}$) and let $m \in \mathcal{N}_{0}$ be a point where $X_{0}^{+}=X_{0}^{-}$. Suppose we have a second vector field $\tilde{X}_{\varepsilon}^{+}$(resp. $\tilde{X}_{\varepsilon}^{-}$) generating $\mathcal{N}_{\varepsilon}^{+}$(resp. $\left.\mathcal{N}_{\varepsilon}^{-}\right)$. According to Lemma 3 , the differences $X_{0}^{+}-\tilde{X}_{0}^{+}$and $X_{0}^{-}-\tilde{X}_{0}^{-}$are tangent to $\mathcal{N}_{0}$ and therefore so is $X_{0}^{+}-X_{0}^{-}-\left(\tilde{X}_{0}^{+}-\tilde{X}_{0}^{-}\right)$. Now, if $X_{0}^{+}-X_{0}^{-}$vanishes at $m$, then $\tilde{X}_{0}^{+}-\tilde{X}_{0}^{-}$must be tangent to $\mathcal{N}_{0}$. This is a contradiction and therefore $\tilde{X}_{0}^{+}-\tilde{X}_{0}^{-}$ vanishes at $m$ too.

Definition 6. Let $X_{\varepsilon}^{ \pm}$be vector fields generating $\mathcal{N}_{\varepsilon}^{ \pm}$and let $m \in \mathcal{N}_{0}$ be an infinitesimal intersection of $\mathcal{N}_{\varepsilon}^{+}$and $\mathcal{N}_{\varepsilon}^{-}$. We define the linear operator $D_{m, X_{0}^{ \pm}}: T_{m} \mathcal{N}_{0} \rightarrow T_{m} \mathcal{M}$ by

$$
D_{m, X_{0}^{ \pm}}(Y):=\left[\tilde{Y}, X_{0}^{+}-X_{0}^{-}\right]_{m},
$$

where $\tilde{Y} \in \Gamma(T \mathcal{M})$ is any extension to $\mathcal{M}$ of $\iota_{*} Y$, with $\iota: \mathcal{N}_{0} \hookrightarrow \mathcal{M}$ the inclusion map.

Lemma 7. The operator $D_{m, X_{0}^{ \pm}}$is well-defined, i.e., independent of the choice of the extension $\tilde{Y}$.

Proof. Let $Y \in T_{m} \mathcal{N}_{0}$ be a vector. If $\tilde{Y}$ and $\tilde{Y}^{\prime}$ are two extensions of $\iota_{*} Y$, then the difference $\tilde{Y}^{\prime}-\tilde{Y}$ vanishes at $m$ and we have $\left[\tilde{Y}^{\prime}-\tilde{Y}, X_{0}^{+}-X_{0}^{-}\right]_{m}=0$ since $X_{0}^{+}-X_{0}^{-}$ also vanishes at $m$. The definition of $D_{m, X_{0}^{ \pm}}$is thus independent of the choice of the extension $\tilde{Y}$.

Notice that despite the operator $D_{m, X_{0}^{ \pm}}$depends on the choice of the generating vector fields $X_{\varepsilon}^{ \pm}$, the following notion does not. 
Definition 8. Let $\mathcal{N}_{\varepsilon}^{ \pm}$be families of compact submanifolds. An infinitesimal intersection $m \in \mathcal{N}_{0}$ is called a transverse whenever the space $\operatorname{img} D_{m, X_{0}^{ \pm}}$is transverse to $T_{m} \mathcal{N}_{0}$ in $T_{m} \mathcal{M}$, with $X_{\varepsilon}^{ \pm}$any generating vector fields.

Lemma 9. The previous notion of transversality is well-defined, i.e., independent of the choice of the vector fields generating $\mathcal{N}_{\varepsilon}^{ \pm}$.

Proof. Suppose we have two pairs of vector fields $X_{\varepsilon}^{ \pm}$and $\tilde{X}_{\varepsilon}^{ \pm}$generating $\mathcal{N}_{\varepsilon}^{ \pm}$. The operators $D_{m, \tilde{X}_{0}^{ \pm}}$and $D_{m, X_{0}^{ \pm}}$are then simply related by

$$
D_{m, \tilde{X}_{0}^{ \pm}} Y=D_{m, X_{0}^{ \pm}} Y+\left[\tilde{Y}, X_{0}^{+}-X_{0}^{-}-\left(\tilde{X}_{0}^{+}-\tilde{X}_{0}^{-}\right)\right]_{m} .
$$

According to Lemma 3 , both differences $X_{0}^{+}-\tilde{X}_{0}^{+}$and $X_{0}^{-}-\tilde{X}_{0}^{-}$are tangent to $\mathcal{N}_{0}$ and therefore so is $X_{0}^{+}-X_{0}^{-}-\left(\tilde{X}_{0}^{+}-\tilde{X}_{0}^{-}\right)$. Since $D_{m, \tilde{X}_{0}^{ \pm}}(Y)$ and $D_{m, X_{0}^{ \pm}}(Y)$ are independent of the choice of the extension $\tilde{Y}$, we can choose it to be tangent to $\mathcal{N}_{0}$. Therefore, the Lie bracket is also tangent to $\mathcal{N}_{0}$. This implies that $\operatorname{img} D_{m, X_{0}^{ \pm}}$is transverse to $T_{m} \mathcal{N}_{0}$ iff $\operatorname{img} D_{m, \tilde{X}_{0}^{ \pm}}$is.

We give now an equivalent and convenient criterion for the infinitesimal transversality.

Lemma 10. Let $\mathcal{N}_{\varepsilon}^{ \pm}$be families of compact submanifolds. There exist generating vector fields $X_{\varepsilon}^{ \pm}$such that for any infinitesimal intersection $m \in \mathcal{N}_{0}$, the space img $\left(D_{m, X_{0}^{ \pm}}\right)$does not intersect $T_{m} \mathcal{N}_{0}$. For such $X_{\varepsilon}^{ \pm}, m$ is transverse iff

$$
\operatorname{dim}\left(k e r D_{m, X_{0}^{ \pm}}\right)=2 \operatorname{dim} \mathcal{N}_{0}-\operatorname{dim} \mathcal{M} \text {. }
$$

Proof. First, proceeding as in the proof of Lemma 2 , we reduce to families of submanifolds in a neighbourhood $\mathcal{O}$ of the zero-section of a vector bundle over $\mathcal{N}_{0}$, and we can choose the generating vector fields to be vertical translations. Moreover, if for the evaluation $D_{m, X_{0}^{ \pm}}(Y):=\left[\tilde{Y}, X_{0}^{+}-X_{0}^{-}\right]_{m}$ we choose an extension $\tilde{Y}$ which is a lift of a vector field on $\mathcal{N}_{0}$, then the Lie bracket is vertical. This implies that the intersection $\operatorname{img}\left(D_{m, X_{0}^{ \pm}}\right) \cap T_{m} \mathcal{N}_{0}$ reduces to $\{0\}$. For the second point, we notice that the vector spaces img $\left(D_{m, X_{0}^{ \pm}}\right)$and $T_{m} \mathcal{N}_{0}$ are transverse in $T_{m} \mathcal{M}$ iff the dimension of the intersection $\operatorname{img}\left(D_{m, X_{0}^{ \pm}}\right) \cap T_{m} \mathcal{N}_{0}$ is equal to $\operatorname{dim}\left(\operatorname{img} D_{m, X_{0}^{ \pm}}\right)+\operatorname{dim} T_{m} \mathcal{N}_{0}-$ $\operatorname{dim} T_{m} \mathcal{M}$. Since the intersection is $\{0\}$, the transversality condition amounts to requiring that $\operatorname{dim}\left(\operatorname{img} D_{m, X_{0}^{ \pm}}\right)+\operatorname{dim} T_{m} \mathcal{N}_{0}-\operatorname{dim} T_{m} \mathcal{M}=0$. Using then the fact that $\operatorname{dim}\left(\operatorname{img} D_{m, X_{0}^{ \pm}}\right)=\operatorname{dim} \mathcal{N}_{0}-\operatorname{dim}\left(\operatorname{ker} D_{m, X_{0}^{ \pm}}\right)$, we obtain the claimed expression.

We now state the theorem which shows that the infinitesimal transversality is the good notion for our problem. 
Theorem 11. Let $\mathcal{N}_{\varepsilon}^{ \pm} \subset \mathcal{M}$ be families of compact submanifolds. If $m \in \mathcal{N}_{0}$ is a transverse infinitesimal intersection, then near m there is a smooth family of submanifolds $\Lambda_{\varepsilon}$ with $\Lambda_{0} \subset \mathcal{N}_{0}$ and $\Lambda_{\varepsilon}=\mathcal{N}_{\varepsilon}^{+} \cap \mathcal{N}_{\varepsilon}^{-}$for small enough $\varepsilon \neq 0$.

Proof. The proof consists of four arguments.

- First, applying the Tubular Neighbourhood for $\mathcal{N}_{0}$, we transpose the situation to a compact neighbourhood of the zero-section of a vector bundle $E$ over $\mathcal{N}_{0}$. Denote by $\pi: E \rightarrow \mathcal{N}_{0}$ the projection and $\iota: \mathcal{N}_{0} \rightarrow E$ the inclusion map. For small enough $\varepsilon$, the manifolds $\mathcal{N}_{\varepsilon}^{ \pm}$are the graphs of sections, denoted by $\alpha_{\varepsilon}^{ \pm}: \mathcal{N}_{0} \rightarrow E$, with $\pi \circ \alpha_{\varepsilon}^{ \pm}=\mathbb{I}_{\mathcal{N}_{0}}$, which satisfy $\alpha_{0}^{ \pm}=0$. Then, we choose generating vector fields $X_{\varepsilon}^{ \pm}$ which are vertical and constant on the fibers. In other words, we have $X_{\varepsilon}^{ \pm}=\frac{d \alpha_{\varepsilon}^{ \pm}}{d \varepsilon}$ if we identify the fibres with their tangent space.

- Second, denote by $\mathcal{N}_{\varepsilon}=\left(\phi_{X_{\varepsilon}^{-}}^{\varepsilon}\right)^{-1}\left(\mathcal{N}_{\varepsilon}^{+}\right)$and $\alpha_{\varepsilon}: \mathcal{N}_{0} \rightarrow E$ the associated family of sections. One checks easily that $\mathcal{N}_{\varepsilon}^{+}$and $\mathcal{N}_{\varepsilon}^{-}$intersect transversally at a point $m$ iff $\mathcal{N}_{\varepsilon}$ and $\mathcal{N}_{0}$ do at the point $\left(\phi_{X_{\varepsilon}^{-}}^{\varepsilon}\right)^{-1}(m)$. Moreover, a point $m \in \mathcal{N}_{0}$ is a transverse infinitesimal intersection for $\mathcal{N}_{\varepsilon}^{+}$and $\mathcal{N}_{\varepsilon}^{-}$iff it is so for $\mathcal{N}_{\varepsilon}$ and $\mathcal{N}_{0}$. Indeed, the flow generating $\mathcal{N}_{\varepsilon}$ is $\left(\phi_{X_{\varepsilon}^{-}}^{\varepsilon}\right)^{-1} \circ \phi_{X_{\varepsilon}^{+}}^{\varepsilon}$ whose vector field, denoted by $X_{\varepsilon}$, is equal to $\left(\phi_{X_{\varepsilon}^{-}}^{\varepsilon}\right)_{*}^{-1}\left(X_{\varepsilon}^{+}-X_{\varepsilon}^{-}\right)$. For $\varepsilon=0$, one has simply $X_{0}=X_{0}^{+}-X_{0}^{-}$, which proves that the operators $\left[., X_{0}^{+}-X_{0}^{-}\right]$and $\left[., X_{0}-0\right]$ coincide.

- Then, consider the fibrewise dilation by a constant number $\frac{1}{\varepsilon}$, which is a diffeomorphism of $E$ and leaves the zero-section $\mathcal{N}_{0}$ invariant. This means that $\tilde{\alpha}_{\varepsilon}=\frac{\alpha_{\varepsilon}}{\varepsilon}$ is still a section, and it is smooth with respect to $\varepsilon$ even at $\varepsilon=0$ since $\alpha_{0}=0$. Namely, one has $\tilde{\alpha}_{0}=\left.\frac{d \alpha_{\varepsilon}}{d \varepsilon}\right|_{\varepsilon=0}$ which is nothing but $X_{0}$, provided the fibres are identified with their tangent space. We denote by $\tilde{\mathcal{N}}_{\varepsilon}$ the graph of the section $\tilde{\alpha}_{\varepsilon}$, which is thus a smooth family of manifolds. Since the dilatation is a diffeomorphism for all $\varepsilon \neq 0$, then $\mathcal{N}_{\varepsilon}$ and $\mathcal{N}_{0}$ intersect transversally for all $\varepsilon \neq 0$ iff $\tilde{\mathcal{N}}_{\varepsilon}$ and $\mathcal{N}_{0}$ do. Now, we know from the general transversality theory that if $\tilde{\mathcal{N}}_{0}$ and $\mathcal{N}_{0}$ intersect transversally at some point $m$, then for small enough $\varepsilon$ the intersection of $\tilde{\mathcal{N}}_{\varepsilon}$ and $\mathcal{N}_{0}$ near $m$ is a smooth manifold depending smoothly on $\varepsilon$.

- Finally, we show that if $m \in \mathcal{N}_{0}$ is a "transverse infinitesimal intersection" of $\mathcal{N}_{\varepsilon}$ and $\mathcal{N}_{0}$ in the sense of Definition 8, then it is actually a transverse intersection (in the usual sense) of $\tilde{\mathcal{N}}_{0}$ and $\mathcal{N}_{0}$. This can easily be deduced from the following formula

$$
\left(\tilde{\alpha}_{0}\right)_{*}(Y)=\iota_{*} Y+D_{m, X_{0}} Y
$$

which holds for each $Y \in T_{m} \mathcal{N}_{0}$. To show this formula, we first use $\tilde{\alpha}_{0}=\phi_{X_{0}}^{1} \circ \iota$ and thus $\left(\tilde{\alpha}_{0}\right)_{*}(Y)=\left(\phi_{X_{0}}^{1}\right)_{*} \iota_{*} Y$. Let $\tilde{Y} \in \Gamma(T E)$ be any extension to $E$ of $\iota_{*} Y$, i.e., 
a vector field on $E$ satisfying $\iota_{*} Y_{m}=\tilde{Y}_{m}$. We have

$$
\left(\phi_{X_{0}}^{1}\right)_{*} \tilde{Y}=\tilde{Y}+\int_{0}^{1} \frac{d}{d t}\left(\left(\phi_{X_{0}}^{t}\right)_{*} \tilde{Y}\right) d t
$$

By definition of the Lie bracket, we obtain

$$
\left(\phi_{X_{0}}^{1}\right)_{*} \tilde{Y}=\tilde{Y}+\int_{0}^{1}\left(\phi_{X_{0}}^{t}\right)_{*}\left[\tilde{Y}, X_{0}\right] d t
$$

Let's choose $\tilde{Y}$ to be a lift of a vector field on the base $\mathcal{N}_{0}$. Since $X_{0}$ is vertical, it follows that the Lie bracket $\left[\tilde{Y}, X_{0}\right]$ is also vertical, as well as $\left(\phi_{X_{0}}^{t}\right)_{*}\left[\tilde{Y}, X_{0}\right]$. If $m \in \mathcal{N}_{0}$ is an infinitesimal intersection of $\mathcal{N}_{\varepsilon}$ and $\mathcal{N}_{0}$, i.e., a point where $\tilde{\alpha}_{0}$ vanishes, then the vector field $X_{0}$ vanishes everywhere on the fibre above $m$ and the flow $\phi_{X_{0}}^{t}$ restricted to this fibre $\mathcal{M}_{q}$ is the identity for all $t$. Thus, at such a point $m$, one has $\int_{0}^{1}\left(\phi_{X_{0}}^{t}\right)_{*}\left[\tilde{Y}, X_{0}\right] d t=\left[\tilde{Y}, X_{0}\right]_{m}=D_{q, X_{0}}(Y)$ which proves the formula (1).

\subsection{Intersections with constraints}

Suppose now that the two families $\mathcal{N}_{\varepsilon}^{ \pm}$are constrained to an intermediate compact submanifold $\mathcal{P}_{\varepsilon}$ for all $\varepsilon$, i.e., $\mathcal{N}_{\varepsilon}^{ \pm} \subset \mathcal{P}_{\varepsilon} \subset \mathcal{M}$, where $\mathcal{P}_{\varepsilon}$ is a smooth family of submanifolds of codimension at least 1 . The submanifolds $\mathcal{N}_{\varepsilon}^{ \pm}$are thus in no way transverse in $\mathcal{M}$ but they may be so in $\mathcal{P}_{\varepsilon}$ if an appropriate infinitesimal transversality condition is satisfied, as we prove in Theorem 15. But first of all, we prove the following.

Lemma 12. Let $\mathcal{N}_{\varepsilon}^{ \pm} \subset \mathcal{P}_{\varepsilon} \subset \mathcal{M}$ be two families of constrained compact submanifolds. There exist generating vector fields $X_{\varepsilon}^{ \pm}$which generate $\mathcal{P}_{\varepsilon}$ in the same time.

Proof. First, let $\psi_{\varepsilon}$ be a flow generating the family $\mathcal{P}_{\varepsilon}$ and consider the families $\tilde{\mathcal{N}}_{\varepsilon}^{ \pm}:=$ $\psi_{\varepsilon}^{-1}\left(\mathcal{N}_{\varepsilon}^{ \pm}\right)$. These families satisfy $\tilde{\mathcal{N}}_{0}^{ \pm}=\mathcal{N}_{0}$ and they are included in the fixed manifold $\mathcal{P}_{0}$ since $\psi_{\varepsilon}\left(\mathcal{P}_{0}\right)=\mathcal{P}_{\varepsilon}$. Therefore, there exist generating flows for $\tilde{\mathcal{N}}_{\varepsilon}^{ \pm}$inside $\mathcal{P}_{0}$, i.e., families of diffeomorphisms $\varphi_{\varepsilon}^{ \pm}: \mathcal{P}_{0} \rightarrow \mathcal{P}_{0}$, with vector fields $Y_{\varepsilon}^{ \pm}$, such that $\varphi_{\varepsilon}^{ \pm}\left(\mathcal{N}_{0}\right)=\tilde{\mathcal{N}}_{\varepsilon}^{ \pm}$ and $Y_{0}^{+}-Y_{0}^{-}$is not tangent to $\mathcal{N}_{0}$. Extending these flows to families of diffeomorphisms on $\mathcal{M}$, we obtain generating flows $\chi_{\varepsilon}^{ \pm}$of $\tilde{\mathcal{N}}_{\varepsilon}^{ \pm}$on $\mathcal{M}$ with the property that $\chi_{\varepsilon}^{ \pm}\left(\mathcal{P}_{0}\right)=\mathcal{P}_{0}$, and with vector fields $Z_{\varepsilon}^{ \pm}$satisfying $Z_{0}^{+}-Z_{0}^{-}$not tangent to $\mathcal{N}_{0}$. This implies that the families $\phi_{\varepsilon}^{ \pm}:=\psi_{\varepsilon} \circ \chi_{\varepsilon}^{ \pm}$generate $\mathcal{N}_{\varepsilon}^{ \pm}$and satisfy $\phi_{\varepsilon}^{ \pm}\left(\mathcal{P}_{0}\right)=\mathcal{P}_{\varepsilon}$.

Definition 13. Let $\mathcal{N}_{\varepsilon}^{ \pm} \subset \mathcal{P}_{\varepsilon} \subset \mathcal{M}$ be two families of constrained compact submanifolds. An infinitesimal intersection $m \in \mathcal{N}_{0}$ is called transverse in the constraint whenever $\operatorname{img} D_{m, X_{0}^{ \pm}}$is transverse to $T_{m} \mathcal{N}_{0}$ in $T_{m} \mathcal{P}_{0}$, i.e.,

$$
\operatorname{img} D_{m, X_{0}^{ \pm}} \oplus T_{m} \mathcal{N}_{0}=T_{m} \mathcal{P}_{0},
$$

with $X_{\varepsilon}^{ \pm}$any generating vector fields. 
To check that this notion is well-defined, one has to verify two facts. First, the image $\operatorname{img} D_{m, X_{0}^{ \pm}}$is in $T_{m} \mathcal{P}_{0}$. Indeed, for any $Y \in T_{m} \mathcal{N}_{0}$ we can choose an extension $\tilde{Y}$ which is tangent to both $\mathcal{N}_{0}$ and $\mathcal{P}_{0}$, hence $D_{m, \tilde{X}_{0}^{ \pm}}(Y)=\left[\tilde{Y}, X_{0}^{+}-X_{0}^{-}\right]_{m}$ lies in $\mathcal{P}_{0}$ since $\tilde{Y}$ and $X_{0}^{+}-X_{0}^{-}$do. Second, this notion of transversality is independent of the choice of the generating vector fields $X_{\varepsilon}^{ \pm}$, as one can check easily following the proof of Lemma 9 ,

As before, we have an equivalent criterion for transverse infinitesimal intersections, in terms of $\operatorname{dim}\left(\operatorname{ker} D_{m, X_{0}^{ \pm}}\right)$. With the help of Lemma12, Lemma10 transposes straightforwardly to the context with constraint, as follows.

Lemma 14. Let $\mathcal{N}_{\varepsilon}^{ \pm} \subset \mathcal{P}_{\varepsilon} \subset \mathcal{M}$ be two families of constrained compact submanifolds. There exist generating vector fields $X_{\varepsilon}^{ \pm}$such that for any infinitesimal intersection $m \in \mathcal{N}_{0}$, the space $\operatorname{img}\left(D_{m, X_{0}^{ \pm}}\right)$does not intersect $T_{m} \mathcal{N}_{0}$. For such $X_{\varepsilon}^{ \pm}, m$ is transverse in the constraint iff

$$
\operatorname{dim}\left(k e r D_{m, X_{0}^{ \pm}}\right)=2 \operatorname{dim} \mathcal{N}_{0}-\operatorname{dim} \mathcal{P}_{0} .
$$

Theorem 15. Let $\mathcal{N}_{\varepsilon}^{ \pm} \subset \mathcal{P}_{\varepsilon} \subset \mathcal{M}$ be two families of constrained compact submanifolds. If $m \in \mathcal{N}_{0}$ is a transverse infinitesimal intersection in the constraint, then in a neighbourhood of $m$ there is a smooth family of submanifolds $\Lambda_{\varepsilon}$ with $\Lambda_{0} \in \mathcal{N}_{0}$ and $\Lambda_{\varepsilon}=\mathcal{N}_{\varepsilon}^{+} \cap \mathcal{N}_{\varepsilon}^{-}$for small enough $\varepsilon \neq 0$.

Proof. Let's choose a flow $\psi_{\varepsilon}$ generating $\mathcal{P}_{\varepsilon}$ and denote by $Z_{\varepsilon}$ its associated vector field. First, one proves that a point $m \in \mathcal{N}_{0}$ is a transverse infinitesimal intersection of $\mathcal{N}_{\varepsilon}^{+}$ and $\mathcal{N}_{\varepsilon}^{-}$in the constraint $\mathcal{P}_{\varepsilon}$ iff it is a transverse infinitesimal intersection of $\psi_{\varepsilon}^{-1}\left(\mathcal{N}_{\varepsilon}^{+}\right)$ and $\psi_{\varepsilon}^{-1}\left(\mathcal{N}_{\varepsilon}^{-}\right)$in the constraint $\mathcal{P}_{0}$, where $\mathcal{P}_{0}$ is understood here as the constant family $\mathcal{P}_{\varepsilon}=\mathcal{P}_{0}$. Indeed, let's define $\tilde{\mathcal{N}}_{\varepsilon}^{ \pm}:=\psi_{\varepsilon}^{-1}\left(\mathcal{N}_{\varepsilon}^{ \pm}\right)$. Suppose that $m$ is a transverse infinitesimal intersection of $\mathcal{N}_{\varepsilon}^{+}$and $\mathcal{N}_{\varepsilon}^{-}$in the constraint $\mathcal{P}_{\varepsilon}$, i.e., $\operatorname{img} D_{m, X_{0}^{ \pm}} \oplus T_{m} \mathcal{N}_{0}=T_{m} \mathcal{P}_{0}$, where $X_{\varepsilon}^{ \pm}$generates $\mathcal{N}_{\varepsilon}^{ \pm}$. The family $\psi_{\varepsilon}^{-1}\left(\mathcal{N}_{\varepsilon}^{ \pm}\right)$is generated by the flow $\psi_{\varepsilon}^{-1} \circ \phi_{X_{\varepsilon}^{ \pm}}^{\varepsilon}$ whose associated vector field $\tilde{X}_{\varepsilon}^{ \pm}$equals to $\left(\psi_{\varepsilon}^{-1}\right)_{*}\left(-Z_{\varepsilon}+X_{\varepsilon}^{ \pm}\right)$. For $\varepsilon=0$, this reduces $\tilde{X}_{0}^{ \pm}=-Z_{0}+X_{0}^{ \pm}$. Consequently, we have $\tilde{X}_{0}^{+}-\tilde{X}_{0}^{-}=X_{0}^{+}-X_{0}^{-}$and thus $D_{m, \tilde{X}_{0}^{ \pm}}=D_{m, X_{0}^{ \pm}}$. Since $\tilde{\mathcal{N}}_{0}=\mathcal{N}_{0}$, we have shown that $\operatorname{img} D_{m, X_{0}^{ \pm}} \oplus T_{m} \mathcal{N}_{0}=T_{m} \mathcal{P}_{0}$ is equivalent to $\operatorname{img} D_{m, \tilde{X}_{0}^{ \pm}} \oplus T_{m} \mathcal{N}_{0}=T_{m} \mathcal{P}_{0}$.

Now, since the families $\tilde{\mathcal{N}}_{\varepsilon}^{ \pm}$lie in the fixed submanifold $\mathcal{P}_{0}$, we can apply Theorem 11 which insures that near $m$ there is a smooth family of submanifolds $\tilde{\Lambda}_{\varepsilon}$ with $\tilde{\Lambda}_{0} \subset \mathcal{N}_{0}$ and $\tilde{\Lambda}_{\varepsilon}=\tilde{\mathcal{N}}_{\varepsilon}^{+} \cap \tilde{\mathcal{N}}_{\varepsilon}^{-}$for small enough $\varepsilon \neq 0$. Applying then the family of diffeomorphisms $\psi_{\varepsilon}$, we obtain the claimed result for the intersections of the families $\mathcal{N}_{\varepsilon}^{ \pm}$.

\subsection{Lagrangian intersections}

Let us suppose now that $\mathcal{M}$ is endowed with a symplectic structure $\omega$ and that the families of submanifolds $\mathcal{N}_{\varepsilon}^{ \pm}$are Lagrangian for all $\varepsilon$. 


\subsubsection{Mel'nikov 1-form for pairs of Lagrangian submanifolds}

Definition 16. Let $\mathcal{N}_{\varepsilon}^{ \pm} \subset \mathcal{M}$ be two families of compact Lagrangian submanifolds. The Mel'nikov 1-form $\beta \in \Omega^{1}\left(\mathcal{N}_{0}\right)$ is defined by

$$
\left.\beta:=\iota^{*}\left(\left(X_{0}^{+}-X_{0}^{-}\right)\right\lrcorner \omega\right),
$$

where $\iota: \mathcal{N}_{0} \hookrightarrow \mathcal{M}$ is the inclusion map and $X_{\varepsilon}^{ \pm}$are any generating vector fields.

Lemma 17. The Mel'nikov 1-form is well-defined, i.e., independent of the choice of $X_{\varepsilon}^{ \pm}$, and it is a closed form, $d \beta=0$.

Proof. First, if $\tilde{X}_{\varepsilon}^{ \pm}$is a second pair of generating vector fields, we know from Lemma 3 that both differences $X_{0}^{+}-\tilde{X}_{0}^{+}$and $X_{0}^{-}-\tilde{X}_{0}^{-}$are tangent to $\mathcal{N}_{0}$, and therefore so is the vector field $Z=X_{0}^{+}-X_{0}^{-}-\left(\tilde{X}_{0}^{+}-\tilde{X}_{0}^{-}\right)$. If we denote by $\beta$ (resp. $\tilde{\beta}$ ) the Mel'nikov 1 -form defined with $X_{\varepsilon}^{ \pm}$(resp. $\left.\tilde{X}_{\varepsilon}^{ \pm}\right)$, we have the relation $\left.\beta=\tilde{\beta}+\iota^{*}(Z\lrcorner \omega\right)$. The second term vanishes since $Z$ is tangent to $\mathcal{N}_{0}$ which is Lagrangian and therefore $\beta=\tilde{\beta}$.

Second, for each $\varepsilon$ the pull-back $\iota^{*}\left(\phi_{X_{\varepsilon}^{ \pm}}^{\varepsilon}\right)^{*} \omega$ vanishes on $\mathcal{N}_{0}$ since $\phi_{X_{\varepsilon}^{ \pm}}^{\varepsilon} \circ \iota\left(\mathcal{N}_{0}\right)=\mathcal{N}_{\varepsilon}^{ \pm}$ and the manifolds $\mathcal{N}_{\varepsilon}^{ \pm}$are Lagrangian. Taking the derivative with respect to $\varepsilon$ and using Cartan's formula together with $d \omega=0$, one obtains $\left.\iota^{*}\left(\phi_{X_{\varepsilon}^{ \pm}}^{\varepsilon}\right)^{*} d\left(X_{\varepsilon}^{ \pm}\right\lrcorner \omega\right)=0$, i.e., $\left.d\left(\iota^{*}\left(\phi_{X_{\varepsilon}^{ \pm}}^{\varepsilon}\right)^{*}\left(X_{\varepsilon}^{ \pm}\right\lrcorner \omega\right)\right)=0$. Then, for $\varepsilon=0$ one has $\left.d\left(\iota^{*}\left(X_{0}^{ \pm}\right\lrcorner \omega\right)\right)=0$ and the difference between the term with $X_{\varepsilon}^{+}$and the one with $X_{\varepsilon}^{-}$gives exactly $d \beta=0$.

In this symplectic context, one can conveniently reformulate the infinitesimal transversality condition in terms of $\beta$ instead of $X_{0}^{+}-X_{0}^{-}$.

Lemma 18. Let $\mathcal{N}_{\varepsilon}^{ \pm} \subset \mathcal{M}$ be two families of compact Lagrangian submanifolds and $\beta$ the Mel'nikov 1-form. A point $m \in \mathcal{N}_{0}$ is an infinitesimal intersection iff $\beta$ vanishes at $m$.

Proof. The "only if" part of the assumption is obvious. In order to prove the "if" part, let us assume that $\beta=0$ at the point $m$. By definition, this means that $\omega\left(X_{0}^{+}-X_{0}^{-}, \iota_{*} Z\right)=0$ for all $Z \in T_{m} \mathcal{N}_{0}$. This implies that $X_{0}^{+}-X_{0}^{-}$is in the $\omega$-orthogonal of $T_{m} \mathcal{N}_{0}$ which is $T_{m} \mathcal{N}_{0}$ itself, since $\mathcal{N}_{0}$ is Lagrangian. But, $X_{0}^{+}-X_{0}^{-}$is by assumption (Lemma 2) never tangent to $\mathcal{N}_{0}$. Therefore $X_{0}^{+}-X_{0}^{-}=0$ at $m$.

Lemma 19. Let $\mathcal{N}_{\varepsilon}^{ \pm} \subset \mathcal{M}$ be two families of compact Lagrangian submanifolds and $\beta$ the Mel'nikov 1-form. If $m \in \mathcal{N}_{0}$ is an infinitesimal intersection, then the derivative $\nabla \beta: T_{m} \mathcal{N}_{0} \times$ $T_{m} \mathcal{N}_{0} \rightarrow \mathbb{R}$ defined by

$$
(\nabla \beta)(Y, Z)=Y(\beta(\tilde{Z})),
$$

with $\tilde{Z} \in \Gamma\left(T \mathcal{N}_{0}\right)$ any extension of $Z$, is a well-defined symmetric bilinear form. 
Proof. Indeed, by definition of the Lie derivative, one has $Y(\beta(\tilde{Z}))=\mathcal{L}_{\tilde{Y}}(\beta(\tilde{Z}))$, where $\tilde{Y}$ is any extension on $\mathcal{N}_{0}$ of $Y$. Then, the Leibniz rule gives $Y(\beta(\tilde{Z}))=$ $\left.\tilde{Z}\lrcorner \mathcal{L}_{\tilde{Y}} \beta+\left(\mathcal{L}_{\tilde{Y}} \tilde{Z}\right)\right\lrcorner \beta$. The second term vanishes at the point $m$ since $\beta$ does. Then, applying Cartan's formula to the first term, we obtain

$$
Y(\beta(\tilde{Z}))=\tilde{Z}\lrcorner(\tilde{Y}\lrcorner d \beta+d(\beta(\tilde{Y}))) .
$$

The first term vanishes since $\beta$ is closed. We thus have $Y(\beta(\tilde{Z}))=Z(\beta(\tilde{Y}))$ which is independent of the choice of the extension $\tilde{Z}$.

We remark that the use of the symbol $\nabla$ is well-justified since the derivative $(\nabla \beta)(Y, Z)$ is easily shown to be equal to $\left(\nabla^{\prime} \beta\right)(\tilde{Y}, \tilde{Z})_{m}$, where $\nabla^{\prime}$ is any covariant derivative and $\tilde{Y}, \tilde{Z}$ are any extensions to $\mathcal{N}_{0}$ of $Y, Z$. The derivative $\nabla \beta$ is related to $D_{m, X_{0}^{ \pm}}$as follows.

Lemma 20. Let $\mathcal{N}_{\varepsilon}^{ \pm} \subset \mathcal{M}$ be two families of compact Lagrangian submanifolds, $X_{\varepsilon}^{ \pm}$generating vector fields and $\beta$ the Mel'nikov 1-form. For any infinitesimal intersection $m \in \mathcal{N}_{0}$, we have the following relation

$$
(\nabla \beta)(Y, Z)=\omega\left(D_{m, X_{0}^{ \pm}}(Y), i_{*} Z\right),
$$

for all $Y, Z \in T_{m} \mathcal{N}_{0}$.

Proof. By definition, one has $(\nabla \beta)(Y, Z)=\mathcal{L}_{\tilde{Y}}(\beta(\tilde{Z}))$, with $\tilde{Y} \in \Gamma\left(T \mathcal{N}_{0}\right)$ (resp. $\tilde{Z} \in$ $\left.\Gamma\left(T \mathcal{N}_{0}\right)\right)$ any extension of $Y$ (resp. $Z$ ). The Leibniz rule gives $\left.Y(\beta(\tilde{Z}))=Z\right\lrcorner \mathcal{L}_{\tilde{Y}} \beta+$ $\left.\left(\mathcal{L}_{\tilde{Y}} \tilde{Z}\right)\right\lrcorner \beta$. The second term vanishes at the point $m$ since $\beta$ does and introducing the definition of $\beta$ in the first term gives $\left.Y(\beta(\tilde{Z}))=Z\lrcorner \mathcal{L}_{\tilde{Y}}\left(\iota^{*}\left(\left(X_{0}^{+}-X_{0}^{-}\right)\right\lrcorner \omega\right)\right)$. If we choose any extension $Y^{\prime}$ on $\mathcal{M}$ of $\iota_{*} \tilde{Y}$, we have $\left.\left.Y(\beta(\tilde{Z}))=Z\right\lrcorner \iota^{*}\left(\mathcal{L}_{Y^{\prime}}\left(\left(X_{0}^{+}-X_{0}^{-}\right)\right\lrcorner \omega\right)\right)$. Using once again the Leibniz rule provides

$$
\left.\left.Y(\beta(\tilde{Z}))=Z\lrcorner \iota^{*}\left(\left[Y^{\prime}, X_{0}^{+}-X_{0}^{-}\right]\right\lrcorner \omega+\left(X_{0}^{+}-X_{0}^{-}\right)\right\lrcorner \mathcal{L}_{Y^{\prime}} \omega\right) .
$$

The second term vanishes at $m$ since $X_{0}^{+}-X_{0}^{-}$does and the first term is precisely $\left.Z\lrcorner \iota^{*}\left(D_{m, X_{0}^{ \pm}}(Y)\right\lrcorner \omega\right)$, i.e., $\omega\left(D_{m, X_{0}^{ \pm}}(Y), \iota_{*} Z\right)$.

This equality has the following corollary.

Lemma 21. Let $\mathcal{N}_{\varepsilon}^{ \pm} \subset \mathcal{M}$ be two families of compact Lagrangian submanifolds and $\beta$ the Mel'nikov 1-form. There exist generating vector fields $X_{\varepsilon}^{ \pm}$such that for any infinitesimal intersection $m \in \mathcal{N}_{0}$, the space img $\left(D_{m, X_{0}^{ \pm}}\right)$does not intersect $T_{m} \mathcal{N}_{0}$. For such $X_{\varepsilon}^{ \pm}$, we have the relation

$$
\operatorname{ker} D_{m, X_{0}^{ \pm}}=\operatorname{ker} \nabla \beta .
$$


Proof. Indeed, thanks to the relation given in Lemma[20, we see that $\operatorname{ker} D_{m, X_{0}^{ \pm}} \subset \operatorname{ker} \nabla \beta$. The converse inclusion $\operatorname{ker} D_{m, X_{0}^{ \pm}} \supset \operatorname{ker} \nabla \beta$ is proved as follows. First, the existence of generating vector fields $X_{\varepsilon}^{ \pm}$with the announced property is proved in 10. Therefore, if $(\nabla \beta)(Y, Z)=0$ for all $Z \in T_{m} \mathcal{N}_{0}$ then $D_{m, X_{0}^{ \pm}}(Y)$ must lie in the $\omega$-orthogonal of $T_{m} \mathcal{N}_{0}$, which is $T_{m} \mathcal{N}_{0}$ itself. But this is a contradiction and therefore $D_{m, X_{0}^{ \pm}}(Y)$ must vanish.

\subsubsection{Constrained intersections for Lagrangian submanifolds}

We suppose now that our Lagrangian submanifolds $\mathcal{N}_{\varepsilon}^{ \pm}$are constrained to an intermediate submanifold $\mathcal{P}_{\varepsilon}$ for all $\varepsilon$, as described on Section 1.2. Thanks to Lemma 21, the criterion given in Lemma 14 transposes straightforwardly to the Lagrangian case, as follows.

Lemma 22. Let $\mathcal{N}_{\varepsilon}^{ \pm} \subset \mathcal{P}_{\varepsilon} \subset \mathcal{M}$ be two families of constrained compact Lagrangian submanifolds and $\beta$ be the Mel'nikov 1-form. Then, an infinitesimal intersection $m$ is transverse in the constraint iff

$$
\operatorname{dim}(\operatorname{ker} \nabla \beta)=\operatorname{codim} \mathcal{P}_{0} .
$$

We can actually say more than this. Indeed, since $\mathcal{P}_{0}$ contains the Lagrangian manifold $\mathcal{N}_{0}$, it must be coisotropic and the associated isotropic foliation $\left(T_{m} \mathcal{P}_{0}\right)^{\perp}$ satisfies $\left(T_{m} \mathcal{P}_{0}\right)^{\perp} \subset T_{m} \mathcal{N}_{0}$ for all $m \in \mathcal{N}_{0}$. Moreover, the dimension of the isotropic foliation is exactly equal to $\operatorname{codim} \mathcal{P}_{0}$. This allows to show Proposition 24 which will be easily deduced from the following lemma.

Lemma 23. Let $\mathcal{N}_{\varepsilon}^{ \pm} \subset \mathcal{M}$ be two families of compact Lagrangian submanifolds and $\beta$ the Mel'nikov 1-form. If $F_{\varepsilon} \in C^{\infty}(\mathcal{M})$ is a family of smooth functions constant on $\mathcal{N}_{\varepsilon}^{+}$and $\mathcal{N}_{\varepsilon}^{-}$for all $\varepsilon$, then the Hamiltonian vector field $X_{F_{0}}$ is tangent to $\mathcal{N}_{0}$ and satisfies

$$
\beta\lrcorner X_{F_{0}}=0
$$

everywhere on $\mathcal{N}_{0}$.

Proof. Let $X_{\varepsilon}^{ \pm}$be vector fields generating the families $\mathcal{N}_{\varepsilon}^{ \pm}$. The Mel'nikov 1-form is related to them by $\left.\beta=\iota^{*}\left(\left(X_{0}^{+}-X_{0}^{-}\right)\right\lrcorner \omega\right)$. By hypothesis, there exists a family of real numbers $c_{\varepsilon}$ such that $F_{\varepsilon} \circ \phi_{X_{\varepsilon}^{ \pm}}^{\varepsilon} \circ \iota=c_{\varepsilon}$ for all $\varepsilon$, where $\iota: \mathcal{N}_{0} \hookrightarrow \mathcal{M}$ is the inclusion map. Taking the derivative with respect to $\varepsilon$, one obtains

$$
\left(\frac{F_{\varepsilon}}{d \varepsilon}+X_{\varepsilon}^{ \pm}\left(F_{\varepsilon}\right)\right) \circ \phi_{X_{\varepsilon}^{ \pm}}^{\varepsilon} \circ \iota=\frac{d c_{\varepsilon}}{d \varepsilon} .
$$

Denoting by a dot the derivatives with respect to $\varepsilon$, one has

$$
\left(\dot{F}_{0}+X_{0}^{ \pm}\left(F_{0}\right)\right) \circ \iota=\dot{c}_{0}
$$


since $\left(\phi_{X_{\varepsilon}^{ \pm}}^{\varepsilon}\right)_{\varepsilon=0}=\mathbb{I}$. The difference between the term with $X_{0}^{+}$and the one with $X_{0}^{-}$ gives simply $\left(X_{0}^{+}-X_{0}^{-}\right)\left(F_{0}\right) \circ \iota=0$. Now, by definition of the Hamiltonian vector field $X_{F_{0}}$, the function $\left(X_{0}^{+}-X_{0}^{-}\right)\left(F_{0}\right)$ is equal to $\omega\left(X_{0}^{+}-X_{0}^{-}, X_{F_{0}}\right)$. Moreover, $X_{F_{0}}$ is tangent to $\mathcal{N}_{0}$ because at each $m \in \mathcal{N}_{0}$, the Lagrangian space $T_{m} \mathcal{N}_{0}$ is included in $\operatorname{ker}\left(d F_{0}\right)_{m}$. This implies that $\omega\left(X_{0}^{+}-X_{0}^{-}, X_{F_{0}}\right)$ is simply $\beta\left(X_{F_{0}}\right)$ and the result follows.

Proposition 24. Let $\mathcal{N}_{\varepsilon}^{ \pm} \subset \mathcal{P}_{\varepsilon} \subset \mathcal{M}$ be two families of constrained compact Lagrangian submanifolds and $\beta$ the Mel'nikov 1-form. Then an infinitesimal intersection $m \in \mathcal{N}_{0}$ is transverse in the constraint iff

$$
\operatorname{ker} \nabla \beta=\left(T_{m} \mathcal{P}_{0}\right)^{\perp}
$$

Proof. First, there exist $p$ smooth families of linearly independent functions $F_{\varepsilon}^{(1)}, \ldots, F_{\varepsilon}^{(p)} \in$ $C^{\infty}(\mathcal{M})$, where $p=\operatorname{codim} \mathcal{P}_{\varepsilon}$, such that in a neighbourhood of $m$ the manifold $\mathcal{P}_{\varepsilon}$ is given by the common level set $\mathcal{P}_{\varepsilon}=\left\{m \mid F_{\varepsilon}^{(1)}(m)=c_{\varepsilon}^{(1)}, \ldots, F_{\varepsilon}^{(p)}(m)=c_{\varepsilon}^{(p)}\right\}$, where $c_{\varepsilon}^{(j)}$ are families of real numbers. Applying the preceding lemma, we obtain that $X_{F_{0}^{(j)}}$ is tangent to $\mathcal{N}_{0}$ and satisfies $\left.\beta\right\lrcorner X_{F_{0}^{(j)}}=0$ everywhere on $\mathcal{N}_{0}$, for each $j=1 . . p$. Now, at each $m \in \mathcal{N}_{0}$ the vectors $X_{F_{0}^{(j)}}$ form a basis of $\left(T_{m} \mathcal{P}_{0}\right)^{\perp}$. This implies that $\left(T_{m} \mathcal{P}_{0}\right)^{\perp} \subset$ $\operatorname{ker} \beta$ everywhere on $\mathcal{N}_{0}$. Therefore, if $m$ is an infinitesimal intersection, then for each $Z \in\left(T_{m} \mathcal{P}_{0}\right)^{\perp} \subset T_{m} \mathcal{N}_{0}$, one has $(\nabla \beta)(Y, Z)=Y(\beta(\tilde{Z}))=0$ since we can choose the extension $\tilde{Z}$ to be everywhere in $\left(T_{m} \mathcal{P}_{0}\right)^{\perp}$. For such a $\tilde{Z}$, one has $\beta(\tilde{Z})$ everywhere and therefore $(\nabla \beta)(Y, Z)=0$ for all $Y$. This shows that $\left(T_{m} \mathcal{P}_{0}\right)^{\perp} \subset \operatorname{ker} \nabla \beta$. This inclusion together with the transversality condition $\operatorname{dim}(\operatorname{ker} \nabla \beta)=\operatorname{dim}\left(T_{m} \mathcal{P}_{0}\right)^{\perp}$ of Lemma 14 proves the result.

\section{The Mel'nikov 1-form}

In the previous section, we developed tools to deal with pairs $\mathcal{N}_{\varepsilon}^{ \pm}$of families of Lagrangian submanifolds, with the same limit $\mathcal{N}_{0}:=\mathcal{N}_{0}^{+}=\mathcal{N}_{0}^{-}$and constrained for all $\varepsilon$ to a submanifold $\mathcal{P}_{\varepsilon}$. We will now use these tools to deal with the situation where $\mathcal{N}_{\varepsilon}^{ \pm}$ are respectively the stable and unstable manifolds of transversally hyperbolic periodic orbits of a given Hamiltonian on $\mathcal{M}$. The Mel'nikov 1-form introduced in Definition 16 allows us to detect the presence of intersections of $\mathcal{N}_{\varepsilon}^{-}$and $\mathcal{N}_{\varepsilon}^{+}$, i.e., heteroclinic orbits between the two periodic orbits. After setting precisely the heteroclinic and homoclinic situation we will deal with, we show that the Mel'nikov 1-form admit an integral expression whenever the Hamiltonian is completely integrable. This integral is unfortunately not convergent in general and needs a prescription on the way we take the limit. Nevertheless, we consider two cases in which this integral is convergent. In particular, this encompasses the historical Mel'nikov setup (time-periodic perturbation of time-independent systems) which is presented as a conclusion of this paper. 


\subsection{Heteroclinic and homoclinic motions}

\subsubsection{Stable and unstable manifolds of transversally hyperbolic orbits}

Suppose the dimension of $\mathcal{M}$ is at least 4 . Let $H \in C^{\infty}(\mathcal{M})$ be a Hamiltonian and denote by $X_{H}$ its vector field and by $\phi^{t}$ its flow. We recall here some basic facts about stable and unstable manifolds of transversally hyperbolic periodic orbit and refer e.g. to [1] for more details.

Definition 25. A $\tau$-periodic orbit $\gamma$ of $X_{H}$ is called (transversally) non-degenerate whenever the eigenvalue $\lambda=1$ of the derivative map $\phi_{*}^{\tau}$ at some point $m \in \gamma$ has multiplicity 2. If moreover the other eigenvalues do not lie on the unit circle, $\gamma$ is called (transversally) hyperbolic.

Note that the eigenvalues of the map $\phi_{*}^{\tau}$ always come in pairs $\left(\lambda, \lambda^{-1}\right)$ since $\phi^{\tau}$ is a symplectic map. On the other hand, at the point $m$ the vector $X_{H}$ itself is obviously an eigenvector with eigenvalue 1 .

It is well-known that the nondegeneracy condition implies that such a periodic orbit always arises within an orbit-cylinder $\Gamma$, i.e., there is an embedding $\Gamma: S^{1} \times[a, b] \rightarrow \mathcal{M}$, with $H(\gamma) \in[a, b]$, such that for each $E \in[a, b]$, the circle $\gamma_{E}=\Gamma\left(S^{1} \times\{E\}\right)$ is a closed orbit of $X_{H}$ and moreover $\Gamma$ is transversal to the energy surfaces $\{m ; H(m)=E\}$.

Furthermore, the hyperbolicity of a periodic orbit $\gamma$ implies the existence of the socalled stable and unstable manifolds. The stable (resp. unstable) manifold is the set, denoted by $\mathcal{N}^{+}$(resp. $\mathcal{N}^{-}$) of points $m \in \mathcal{M}$ such that $\phi^{t}(m)$ tends to the limit cycle $\gamma$ when $t \rightarrow+\infty$ (resp. $t \rightarrow+\infty$ ). One is usually obliged to distinguish between the local and the global (un)stable manifolds. Indeed, the hyperbolicity condition implies that in a neighbourhood of $\gamma$, there exist two embedded Lagrangian submanifolds $\mathcal{N}_{\text {loc }}^{+}$ and $\mathcal{N}_{l o c}^{-}$, called the local stable and unstable manifolds, whose intersection is exactly $\gamma$. The global stable and unstable manifolds are then obtained from the local ones by applying the flow $\phi^{t}$ for all $t$, and in general they are injectively immersed in $\mathcal{M}$ in a very complicated way.

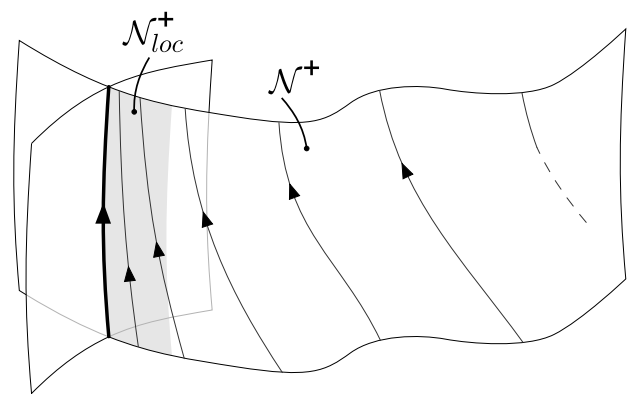

In the sequel, we will need to focus on a compact part of the stable and unstable manifolds. For this purpose, we define the following.

Definition 26. For each $T>0$, we define the compact manifold $\mathcal{N}_{T}^{ \pm}:=\phi^{\mp T}\left(\overline{\mathcal{N}_{\text {loc }}^{ \pm}}\right)$. 
These manifolds depend of course on the choice of the local manifolds $\mathcal{N}_{\text {loc }}^{ \pm}$, but they satisfy $\mathcal{N}_{T}^{ \pm} \subset \mathcal{N}_{T^{\prime}}^{ \pm}$for all $T<T^{\prime}$, and $\lim _{T \rightarrow+\infty} \mathcal{N}_{T}^{ \pm}=\mathcal{N}^{ \pm}$.

We remark that in dimension 4, the manifolds $\mathcal{N}_{\text {loc }}^{ \pm}$(and thus $\mathcal{N}_{T}^{ \pm}$as well) may be such that $\mathcal{N}_{\text {loc }}^{ \pm} \backslash \gamma$ has two connected components, say $\mathcal{N}_{1}^{ \pm}$and $\mathcal{N}_{2}^{ \pm}$, as depicted on the left hand side below.
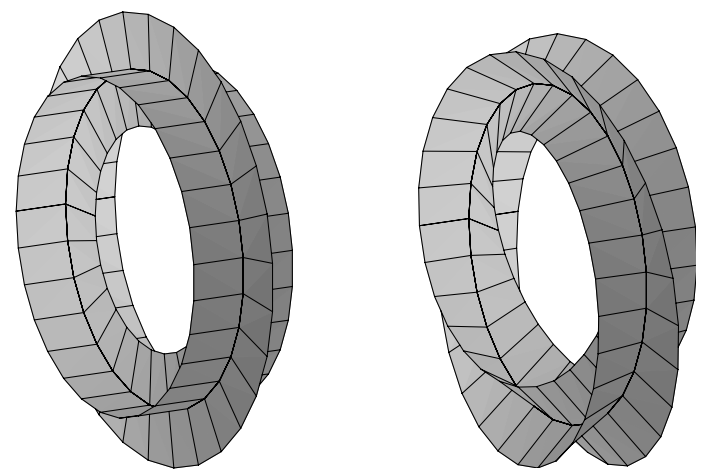

In that case, $\mathcal{N}_{T}^{ \pm}$will rather denote $\phi^{\mp T}\left(\overline{\mathcal{N}_{j}^{ \pm}}\right)$with the choice of a connected component ${ }^{2} j=1$ or 2 . As well, $\mathcal{N}^{ \pm}$will denote one component of the (un)stable manifold rather than the full manifold. In higher dimensions, this distinction is irrelevant since the manifolds $\mathcal{N}_{\text {loc }}^{ \pm} \backslash \gamma$ are connected.

\subsubsection{Heteroclinic and homoclinic motions}

Let $H_{0} \in C^{\infty}(\mathcal{M})$ be a Hamiltonian which admits two hyperbolic periodic orbits $\gamma_{0}^{+}$ and $\gamma_{0}^{-}$, and denote by $\phi^{t}$ its flow. As explained in the previous section, the orbit $\gamma_{0}^{+}$ (resp. $\gamma_{0}^{-}$) has a stable and an unstable manifold $\mathcal{N}^{ \pm}\left(\gamma_{0}^{+}\right)$(resp. $\mathcal{N}^{ \pm}\left(\gamma_{0}^{-}\right)$). Let us focus now on the two manifolds $\mathcal{N}^{+}\left(\gamma_{0}^{+}\right)$and $\mathcal{N}^{-}\left(\gamma_{0}^{-}\right)$. Any point $m \in \mathcal{N}^{+}\left(\gamma_{0}^{+}\right) \cap \mathcal{N}^{-}\left(\gamma_{0}^{-}\right)$ is called a heteroclinic point and its orbit $t \rightarrow \phi^{t}(m)$ is a heteroclinic orbit between $\gamma_{0}^{-}$ and $\gamma_{0}^{+}$, i.e., it tends to $\gamma_{0}^{-}$(resp. $\gamma_{0}^{+}$) when $t \rightarrow-\infty$ (resp. $t \rightarrow+\infty$ ). When the two periodic orbits coincide $\gamma_{0}=\gamma_{0}^{+}=\gamma_{0}^{-}$, then any point in $\mathcal{N}^{+}\left(\gamma_{0}\right) \cap \mathcal{N}^{-}\left(\gamma_{0}\right)$ is called a homoclinic point and its orbit $t \rightarrow \phi^{t}(m)$ is a homoclinic orbit, i.e., it tends to $\gamma_{0}$ when $t \rightarrow \pm-\infty$.

In general, the two manifolds $\mathcal{N}^{+}\left(\gamma_{0}^{+}\right)$and $\mathcal{N}^{-}\left(\gamma_{0}^{-}\right)$have no reason to coincide and the set of heteroclinic points may be very complicated.

\footnotetext{
${ }^{2}$ See e.g. [4] for a study of 4-dimensional completely integrable systems with transversally hyperbolic periodic orbits.
} 


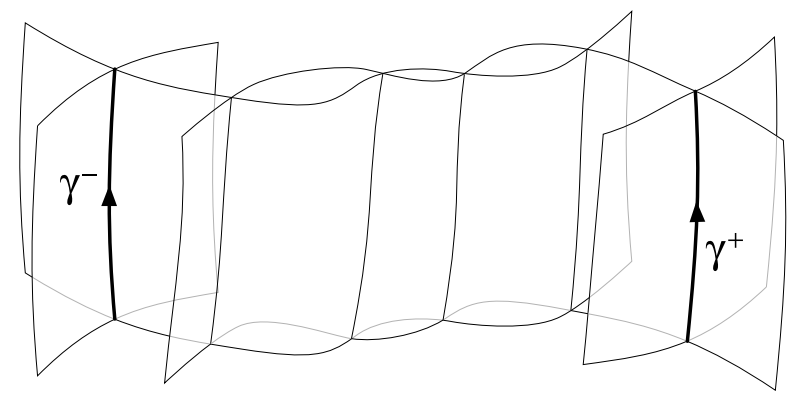

Nevertheless, Mel'nikov's theory deals precisely with perturbations $H_{\varepsilon}$ of a Hamiltonian $H_{0}$ with two hyperbolic periodic orbits $\gamma_{0}^{+}$and $\gamma_{0}^{-}$such that the closure of the stable manifolds $\mathcal{N}^{+}\left(\gamma_{0}^{+}\right)$do coincide with the closure of the unstable manifold $\mathcal{N}^{-}\left(\gamma_{0}^{-}\right)$. We introduce the following notation.

Definition 27. We define $\mathcal{N}_{0}^{ \pm}:=\mathcal{N}^{ \pm}\left(\gamma_{0}^{ \pm}\right)$. In the 4-dimensional case, $\mathcal{N}^{ \pm}\left(\gamma_{0}^{ \pm}\right)$denotes one connected component of the (un)stable manifold, as explained in the previous section.

Definition 28. From now on, we focus on the following two situations :

- Heteroclinic situation. We suppose that the Hamiltonian $H_{0}$ admits two hyperbolic periodic orbits $\gamma_{0}^{+}$and $\gamma_{0}^{-}$. Moreover, we suppose that the closure of the stable manifold $\mathcal{N}_{0}^{+}$of $\gamma_{0}^{+}$coincides with the closure of the unstable manifold $\mathcal{N}_{0}^{-}$ of $\gamma_{0}^{-}$, and we denote by $\mathcal{N}_{0}=\overline{\mathcal{N}_{0}^{+}}=\overline{\mathcal{N}_{0}^{-}}$this heteroclinic manifold.

- Homoclinic situation. We suppose that the Hamiltonian $H_{0}$ admits one hyperbolic periodic orbit $\gamma_{0}$. Moreover, we suppose that the closures of its stable and unstable manifolds coincide, and we denote by $\mathcal{N}_{0}=\overline{\mathcal{N}_{0}^{+}}=\overline{\mathcal{N}_{0}^{-}}$this homoclinic manifold.

Despite in general the manifolds $\mathcal{N}_{0}^{+}$and $\mathcal{N}_{0}^{-}$are immersed in $\mathcal{M}$ in a complicated way, when $\overline{\mathcal{N}_{0}^{+}}$and $\overline{\mathcal{N}_{0}^{-}}$coincide, then they have the following nice form.

Lemma 29. There is a time $\kappa$ such that $\mathcal{N}_{0, \kappa}^{+} \cup \mathcal{N}_{0, \kappa}^{-}=\mathcal{N}_{0}$, where $\mathcal{N}_{0, \kappa}^{ \pm}$denote the manifolds defined in Definition [26] Moreover, one has

$$
\lim _{\kappa \rightarrow+\infty} \mathcal{N}_{0, \kappa}^{+} \cap \mathcal{N}_{0, \kappa}^{-}=\mathcal{N}_{0} .
$$

One the picture below, the thin lines represent $\mathcal{N}_{0}$ while the thick ones represent $\mathcal{N}_{0, \kappa}^{ \pm}$.

\begin{tabular}{|l|l|}
\cline { 2 - 3 } \multicolumn{1}{c|}{ Small $\kappa$} & Heteroclinic \\
\hline large $\kappa$ & \\
\hline
\end{tabular}




\subsection{Heteroclinic/homoclinic orbits and the Mel'nikov 1-form}

\subsubsection{Splitting of heteroclinic/homoclinic Lagrangian submanifolds and the Mel'nikov 1 -form}

Let $H_{0} \in C^{\infty}(\mathcal{M})$ be a Hamiltonian either in the heteroclinic or in the homoclinic situation (see Definition 28) and let $\mathcal{N}_{0, \kappa}^{ \pm}$be the corresponding manifolds for a chosen large $\kappa>0$. A very important consequence of the hyperbolicity of the periodic orbits $\gamma_{0}^{ \pm}$is that the system is structurally stable [8]. This means that if $H_{\varepsilon} \in C^{\infty}(\mathcal{M})$ is a perturbation of $H_{0}$, then in a neighbourhood of $\gamma_{0}^{ \pm}$there is an hyperbolic periodic orbit $\gamma_{\varepsilon}^{ \pm}$of $H_{\varepsilon}$ $\varepsilon$-close to $\gamma_{0}^{ \pm}$. Moreover, the stable and unstable manifolds of $H_{\varepsilon}$ are $\varepsilon$-close to those of $\gamma_{\varepsilon}^{ \pm}$. Actually, the smoothness of $H_{\varepsilon}$ with respect to $\varepsilon$ implies the smoothness of $\gamma_{\varepsilon}^{ \pm}$and $\mathcal{N}_{\varepsilon}^{ \pm}$.

Restricting to a compact part as in Definition 26, we thus have two families of periodic orbits $\gamma_{\varepsilon}^{ \pm}$together with two families of manifolds $\mathcal{N}_{\varepsilon, \kappa}^{ \pm}$and we want to detect at first order in $\varepsilon$ the intersections $\mathcal{N}_{\varepsilon, \kappa}^{+} \cap \mathcal{N}_{\varepsilon, \kappa}^{-}$for small $\varepsilon$ using the Mel'nikov 1-form defined in the first section. Unfortunately, we are not strictly speaking in the Mel'nikov setting since $\mathcal{N}_{0, \kappa}^{+}$and $\mathcal{N}_{0, \kappa}^{-}$do not coincide exactly. Nevertheless, for large $\kappa$ the intersection $\mathcal{N}_{0, \kappa}^{+} \cap \mathcal{N}_{0, \kappa}^{-}$tends to $\mathcal{N}_{0}$. In order to avoid an useless complexification of the notations, we will make a slight misuse of notations by using the Mel'nikov 1-form $\beta \in \Omega^{1}\left(\mathcal{N}_{0}\right)$ for the families $\mathcal{N}_{\varepsilon, \kappa}^{ \pm}$, being implicitly understood that it is defined only inside the intersection $\mathcal{N}_{0, \kappa}^{+} \cap \mathcal{N}_{0, \kappa}^{-}$, i.e., away from the periodic orbits $\gamma_{0}^{ \pm}$.

Since the system is Hamiltonian, the families $\mathcal{N}_{\varepsilon, \kappa}^{ \pm}$are included in a level set $H_{\varepsilon}=$ $\operatorname{cst}(\varepsilon)$ for each $\varepsilon$ and we are thus in the constrained setting developed in Section 1.3.2. We know from there that the transverse infinitesimal intersections of $\mathcal{N}_{\varepsilon, \kappa}^{+}$and $\mathcal{N}_{\varepsilon, \kappa}^{-}$are slightly deformed by the perturbation, and this shows that the Mel'nikov 1-form is the right object for detecting the existence of some of the intersections of $\mathcal{N}_{\varepsilon, \kappa}^{+}$and $\mathcal{N}_{\varepsilon, \kappa}^{-}$when $\varepsilon \neq 0$, i.e., heteroclinic points between $\gamma_{\varepsilon}^{-}$and $\gamma_{\varepsilon}^{+}$.

\subsubsection{An invariance property of the Mel'nikov 1- form}

Let $H_{\varepsilon} \in C^{\infty}(\mathcal{M})$ be a perturbation of a Hamiltonian $H_{0} \in C^{\infty}(\mathcal{M})$ either in the heteroclinic or in the homoclinic situation, and let $\beta \in \Omega^{1}\left(\mathcal{N}_{0}\right)$ be the associated Mel'nikov 1 -form. Lemma[23] says that $\beta\left(X_{H_{0}}\right)=0$ everywhere on $\mathcal{N}_{0}$. This implies that the zeros of the Mel'nikov 1-form come together with their orbit, as explained below.

Lemma 30. If $m \in \mathcal{N}_{0}$ is an infinitesimal intersection, $\beta_{m}=0$, then each point of the orbit $\phi_{X_{H_{0}}}^{t}(m)$ is so. If $m$ is transversal in the constraint then each point of the orbit $\phi_{X_{H_{0}}}^{t}(m)$ is so.

Proof. The first point comes directly from the Cartan's formula $\left.\mathcal{L}_{X_{H_{0}}} \beta=X_{H_{0}}\right\lrcorner d \beta+$ $d\left(\beta\left(X_{H_{0}}\right)\right)$. The first terms vanishes since $\beta$ is closed and the second one vanishes thanks to Lemma 23. The Mel'nikov 1-form is thus invariant by the flow of $X_{H_{0}}$ and the first point is proved. To prove the second one, let us choose an affine connection $\nabla$ such that $\nabla X_{H_{0}}=0$ in the neighbourhood $\mathcal{O}$ of a transversal infinitesimal intersection $m$. This is always possible since $X_{H_{0}}$ does not vanish on $\mathcal{N}_{0}$. We will show that 
$\mathcal{L}_{X_{H_{0}}}(\nabla \beta)=0$ and this will prove the second point. To evaluate $\mathcal{L}_{X_{H_{0}}}(\nabla \beta)(Y, Z)$ at a point $m$, we extend $Y$ and $Z$ to $\mathcal{O}$ in such a way that $\nabla Y=0$ and $\nabla Z=0$. Since $X_{H_{0}}, Y$ and $Z$ are parallel vector fields, they commute with each other. This implies that

$$
\mathcal{L}_{X_{H_{0}}}(\nabla \beta)(Y, Z)=\mathcal{L}_{X_{H_{0}}}((\nabla \beta)(Y, Z))=\mathcal{L}_{X_{H_{0}}}(Y(\beta(Z))) .
$$

The Leibniz rule for the Lie derivative then gives

$$
\mathcal{L}_{X_{H_{0}}}(Y(\beta(Z)))=Y\left(\left(\mathcal{L}_{X_{H_{0}}} \beta\right)(Z)\right)
$$

and this vanishes as we have shown earlier. The $(2,0)$-tensor field $\nabla \beta$ is thus invariant by the flow of $X_{H_{0}}$. According to Proposition 24, a point $m$ is a transversal infinitesimal intersection, iff ker $\nabla \beta$ is exactly the line generated by $X_{H_{0}}$. Now, since $X_{H_{0}}$ and $\nabla \beta$ are invariant by the flow of $X_{H_{0}}$, then we have

$$
\left.\left.\left(\phi_{X_{H_{0}}}^{-t}\right)^{*}\left(X_{H_{0}}\right\lrcorner \nabla \beta\right)_{m}=\left(X_{H_{0}}\right\lrcorner \nabla \beta\right)_{\phi_{X_{H_{0}}}^{t}(m)} .
$$

This means that ker $\nabla \beta$ at $m$ is generated by $X_{H_{0}}$ iff it is so at each point of the orbit $\phi_{X_{H_{0}}}^{t}(m)$.

\subsubsection{Mel'nikov potentials}

Lemma 31. The Mel'nikov 1-form $\beta$ is exact. Any primitive, i.e., any function $L \in C^{\infty}\left(\mathcal{N}_{0}\right)$ with $\beta=d L$, is called a Mel'nikov potential.

Proof. We already know from Lemma 17 that $\beta$ is closed. Therefore, it is exact if $\int_{\gamma} \beta=0$ for cycles $\gamma$ generating the homology group $H_{1}\left(\mathcal{N}_{0}\right)$. Actually, we see from Definition 16 that $\beta$ is a difference $\beta=\beta^{+}-\beta^{-}$, where the $\beta^{ \pm}$are closed 1-forms defined on $\mathcal{N}_{0} \backslash \gamma_{0}^{\mp}$. Now, the manifolds $\mathcal{N}_{0} \backslash \gamma_{0}^{\mp}$ are diffeomorphic to $S^{1} \times \mathbb{R}^{d-1}$ (or $S^{1} \times \mathbb{R}^{+}$for some 4-dimensional systems, as explained at the end of Section 2.1.1). Their homology is thus generated precisely by the cycle $\gamma_{0}^{ \pm}$. But these are trajectories of $H_{0}$. Therefore, one has

$$
\int_{\gamma_{0}^{ \pm}} \beta^{ \pm}=\frac{1}{\tau^{ \pm}} \int_{0}^{\tau^{ \pm}} \beta^{ \pm}\left(X_{H_{0}}\right) \circ \phi_{X_{H_{0}}}^{s}\left(m_{0}\right) d s,
$$

with $m_{0}$ any point on $\gamma_{0}^{ \pm}$and $\tau^{ \pm}$the period of the orbit $\gamma_{0}^{ \pm}$. Using Lemma 23, we conclude that $\int_{\gamma_{0}^{ \pm}} \beta=0$ and therefore $\beta$ is exact.

Despite this apparently pleasant property, we will not use Mel'nikov potentials, for several reasons. First of all, the object which parameterises the deformations of the Lagrangian (stable and unstable) submanifolds is really a closed 1-form and not its primitive. Second, the heteroclinic points are detected by the zeros of $\beta$, i.e., the critical points of a primitive $L$. Thus, in any case, one has to compute the derivative of $L$. Third, it might happen that $\beta$ admits a nice integral expression, but $L$ does not, as we explain later in Section 2.3.5. 


\subsection{Integral expression in the CI Case}

Consider a Hamiltonian $H_{0} \in C^{\infty}(\mathcal{M})$ either in the heteroclinic or in the homoclinic situation and let $H_{\varepsilon} \in C^{\infty}(\mathcal{M})$ be a perturbation. The definition of the Mel'nikov 1-form associated with the deformed stable and unstable manifolds does actually not take into account the dynamical character of these manifolds. But, we will now show that there is an integral expression for the contraction $\beta\left(X_{A}\right)$, when $A$ is any conserved quantity, i.e., a function on $\mathcal{M}$ satisfying $\left\{A, H_{0}\right\}=0$.

When such a conserved quantity $A$ exists, the dynamical character of the system allows one to give an integral expression for $\beta\left(X_{A}\right)$, which corresponds in some special cases to the object called Mel'nikov function presented in the literature. Unfortunately, in the general case this integral does not converge and one has to give a prescription to make it converge. We explain this issue in Section 2.3.2. Nevertheless, there are two cases where the integral converges. They are discussed in Sections 2.3.3 and 2.3.4. First, we describe the situation where the perturbation is critical on the orbits $\gamma_{0}^{+}$and $\gamma_{0}^{-}$. Finally, we consider the case when the conserved quantity $A$ is critical on $\gamma_{0}^{+}$and $\gamma_{0}^{-}$. We notice that in order to describe completely $\beta$, one needs to have $d$ Hamiltonian vector fields $X_{A_{1}}, \ldots, X_{A_{d}}$ tangent to $\mathcal{N}_{0}$ and linearly independent. This arises precisely when $H_{0}$ is completely integrable and the $A_{j}$ 's are the components of a momentum map. In Section 2.3.4 we explain how many linearly independent $A_{j}$ 's critical on the orbits $\gamma_{0}^{+}$ and $\gamma_{0}^{-}$one can have.

\subsubsection{Momentum maps in presence of transversally hyperbolic periodic orbits}

The presence of a hyperbolic periodic orbit for a Hamiltonian $H$ implies certain properties for its conserved quantities $A,\{A, H\}=0$, as follows.

Proposition 32. Suppose $H$ has a transversally hyperbolic periodic orbit $\gamma$. Then, each conserved quantity $A$ is constant on the stable and unstable manifolds $\mathcal{N}^{ \pm}(\gamma)$, i.e., $A\left(\mathcal{N}^{+}(\gamma)\right)=$ $A\left(\mathcal{N}^{-}(\gamma)\right)=A(\gamma)$, and its vector field $X_{A}$ is tangent to $\mathcal{N}^{+}(\gamma)$ and $\mathcal{N}^{-}(\gamma)$. Moreover, there is a constant $c(A)$ such that $X_{A}=c(A) X_{H}$ at each point of $\gamma$.

Proof. The commutation relation $\{A, H\}=0$ implies that the orbits of $X_{H}$ are included in the level sets $\{m, A(m)=a\}, a \in \mathbb{R}$. In particular one has $\gamma \subset\{m, A(m)=a\}$ for a. Moreover, by definition, for each point $m$ on the stable manifold $\mathcal{N}^{+}(\gamma)$, one has $\phi_{X_{H}}^{t}(m) \rightarrow \gamma$, when $t \rightarrow+\infty$. Since the function $A$ is constant on the trajectories of $X_{H}$, we must have $A(m)=A \circ \phi_{X_{H}}^{t}(m)$ and the limit $t \rightarrow+\infty$ yields $A(m)=A(\gamma)$ for each $m \in \mathcal{N}^{+}(\gamma)$, i.e., $A$ is constant on $\mathcal{N}^{+}(\gamma)$. A similar argument shows that $A$ is also constant on $\mathcal{N}^{-}(\gamma)$. Since $\mathcal{N}^{ \pm}(\gamma)$ is Lagrangian, the inclusion $T_{m} \mathcal{N}^{ \pm}(\gamma) \subset \operatorname{ker} d A_{m}$ at the point $m \in \mathcal{N}^{ \pm}(\gamma)$ is equivalent by duality to $X_{A} \in T_{m} \mathcal{N}^{ \pm}(\gamma)$. Now, at each point $m \in \gamma$, the intersection $T_{m} \mathcal{N}^{-}(\gamma) \cap T_{m} \mathcal{N}^{+}(\gamma)$ is exactly $T_{m} \gamma$ and therefore $X_{A} \in T_{m} \gamma$. Consequently, there is a function $c: \gamma \rightarrow \mathbb{R}$ such that one has the relation $X_{A}=c(m) X_{H}$, at $m \in \gamma$. Moreover, $X_{A}$ is invariant under the flow of $X_{H}$, since $\{H, A\}=0$ and thus $\mathcal{L}_{X_{H}} X_{A}=0$. This implies that $c(m)$ is independent of $m$. 
According to Proposition 32, if $H_{0}$ admits a momentum map $\mathbf{A}=\left(A_{1}, \ldots, A_{d}\right)$ which is regular on $\mathcal{N}_{0} \backslash\left(\gamma_{0}^{+} \cup \gamma_{0}^{-}\right)$, then the Hamiltonian vector fields $X_{A_{1}}, \ldots, X_{A_{d}}$ form a basis of $T_{m} \mathcal{N}_{0}$ at each point $m \in \mathcal{N}_{0} \backslash\left(\gamma_{0}^{+} \cup \gamma_{0}^{-}\right)$. Therefore, the Mel'nikov 1-form $\beta \in \Omega^{1}\left(\mathcal{N}_{0}\right)$ associated to any perturbation $H_{\varepsilon}$ is fully understood whenever one is able to compute the evaluations $\beta\left(X_{A_{1}}\right), \ldots, \beta\left(X_{A_{d}}\right)$.

\subsubsection{Integral expression with prescription}

Thanks to Proposition 32, the vector field $X_{A}$ associated to any conserved quantity $A$ is tangent to the heteroclinic/homoclinic manifold $\mathcal{N}_{0}$, and one can thus evaluate the Mel'nikov 1-form $\beta$ on it. This evaluation can express in terms of an integral involving the first order perturbation $H_{1}=\left.\frac{d H_{\varepsilon}}{d \varepsilon}\right|_{\varepsilon=0^{\prime}}$ as the next theorem shows.

Theorem 33. Let $H_{0} \in C^{\infty}(\mathcal{M})$ be a Hamiltonian either in the heteroclinic or in the homoclinic situation. Let $H_{\varepsilon} \in C^{\infty}(\mathcal{M})$ be a perturbed Hamiltonian and $\beta \in \Omega^{1}\left(\mathcal{N}_{0}\right)$ the associated Mel'nikov 1-form. Then, for any conserved quantity $A \in C^{\infty}(\mathcal{M})$ and any point $m \in \mathcal{N}_{0} \backslash$ $\left(\gamma_{0}^{+} \cup \gamma_{0}^{-}\right)$, one has the following formula

$$
\beta\left(X_{A}\right)_{m}=\left.\frac{d}{d \varepsilon} A\left(m_{\varepsilon}^{+}\right)\right|_{\varepsilon=0}-\left.\frac{d}{d \varepsilon} A\left(m_{\varepsilon}^{-}\right)\right|_{\varepsilon=0}+\lim _{n \rightarrow \infty} \int_{-n \tau_{0}^{-}}^{n \tau_{0}^{+}}\left\{H_{1}, A\right\} \circ \phi_{X_{H_{0}}}^{t}(m) d t,
$$

where $\tau_{\varepsilon}^{ \pm}$is the period of $\gamma_{\varepsilon}^{ \pm}$and the point $m_{\varepsilon}^{ \pm} \in \gamma_{\varepsilon}^{ \pm}$is given by

$$
m_{\varepsilon}^{ \pm}=\lim _{n \rightarrow \infty} \phi_{X_{H_{\varepsilon}}}^{\mp n \tau_{\varepsilon}^{ \pm}} \circ \phi_{X_{\varepsilon}^{ \pm}}^{\varepsilon}(m)
$$

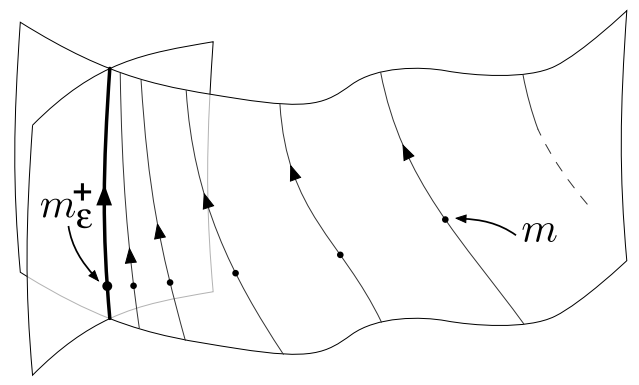

Proof. Fix a constant $\kappa$ large enough for $m$ to belong to $\mathcal{N}_{0, \kappa}^{+} \cap \mathcal{N}_{0, \kappa}^{-}$. Remember that the Mel'nikov 1-form is given in Definition 16 by $\left.\beta=\iota^{*}\left(\left(X_{0}^{+}-X_{0}^{-}\right)\right\lrcorner \omega\right)$, where $X_{\varepsilon}^{+}$(resp. $X_{\varepsilon}^{-}$) generates the stable (resp. unstable) manifold $\mathcal{N}_{\varepsilon, \kappa}^{+}\left(\right.$resp. $\mathcal{N}_{\varepsilon, \kappa}^{-}$) of the orbit $\gamma_{\varepsilon}^{+}$(resp. $\left.\gamma_{\varepsilon}^{-}\right)$. For the evaluation on $X_{A}$, we have to compute both terms $\omega\left(X_{0}^{ \pm}, X_{A}\right)$, which are nothing but $X_{0}^{ \pm}(A)$, i.e., $\left.\frac{d}{d \varepsilon} A \circ \phi_{X_{\varepsilon}^{ \pm}}^{\varepsilon}(m)\right|_{\varepsilon=0}$. Now, by definition of $X_{\varepsilon}^{ \pm}$, the point $\phi_{X_{\varepsilon}^{ \pm}}^{\varepsilon}(m)$ is on $\mathcal{N}_{\varepsilon, \kappa}^{ \pm}$. We will compute an expression for $A \circ \phi_{X_{\varepsilon}^{+}}^{\varepsilon}(m)$ (and later similarly for $\left.A \circ \phi_{X_{\varepsilon}^{-}}^{\varepsilon}(m)\right)$ in terms of an integral which converges uniformly with respect to $\varepsilon$ and then take the derivative. For any time $T$, one has the relation

$$
A \circ \phi_{X_{\varepsilon}^{+}}^{\varepsilon}(m)=A \circ \phi_{X_{H_{\varepsilon}}}^{T} \circ \phi_{X_{\varepsilon}^{+}}^{\varepsilon}(m)-\int_{0}^{T} X_{H_{\varepsilon}}(A) \circ \phi_{X_{H_{\varepsilon}}}^{t} \circ \phi_{X_{\varepsilon}^{+}}^{\varepsilon}(m) d t,
$$


where we have used $\frac{d}{d t} A \circ \phi_{X_{H_{\varepsilon}}}^{t}=X_{H_{\varepsilon}}(A) \circ \phi_{X_{H_{\varepsilon}}}^{t}$. Now, $\phi_{X_{\varepsilon}^{+}}^{\varepsilon}(m)$ is on the stable manifold $\mathcal{N}_{\varepsilon}^{+}$and $\phi_{X_{H_{\varepsilon}}}^{T} \circ \phi_{X_{\varepsilon}^{+}}^{\varepsilon}(m)$ tends to the cycle $\gamma_{\varepsilon}^{+}$when $T \rightarrow \infty$. If one considers the discrete times $T=n \tau_{\varepsilon}^{+}$, with $\tau_{\varepsilon}^{+}$the period of $\gamma_{\varepsilon}^{+}$, then $\phi_{X_{H_{\varepsilon}}}^{n \tau_{\varepsilon}^{+}} \circ \phi_{X_{\varepsilon}^{+}}^{\varepsilon}(m)$ has a limit on $\gamma_{\varepsilon}^{+}$when $n \rightarrow \infty$ and this limit is uniform in $\varepsilon$. Indeed, $\phi_{X_{\varepsilon}^{+}}^{\varepsilon}(m)$ is on the stable manifold of some point $m_{\varepsilon}^{+} \in \gamma_{\varepsilon}^{+}$, i.e., dist $\left(\phi_{X_{H_{\varepsilon}}^{T}}^{T}\left(m_{\varepsilon}^{+}\right), \phi_{X_{H_{\varepsilon}}}^{T} \circ \phi_{X_{\varepsilon}^{+}}^{\varepsilon}(m)\right) \leq C_{\varepsilon} e^{-T \lambda_{\varepsilon}}$ for large $T$. Taking the maximum $C$ over $\varepsilon$ of the constant $C_{\varepsilon}$ and the minimum $\lambda$ of the Liapounov exponent $\lambda_{\varepsilon}$, one obtains

$$
\operatorname{dist}\left(\phi_{X_{H_{\varepsilon}}}^{T}\left(m_{\varepsilon}^{+}\right), \phi_{X_{H_{\varepsilon}}}^{T} \circ \phi_{X_{\varepsilon}^{+}}^{\varepsilon}(m)\right) \leq C e^{-T \lambda}
$$

for all $\varepsilon$ and all $T$. Now, for $T=n \tau_{\varepsilon}^{+}$one has $\phi_{X_{H_{\varepsilon}}}^{n \tau_{\varepsilon}^{+}}\left(m_{\varepsilon}^{+}\right)=m_{\varepsilon}^{+}$and therefore for all positive integers $n$ and all $\varepsilon$, one has

$$
\operatorname{dist}\left(m_{\varepsilon}^{+}, \phi_{X_{H_{\varepsilon}}}^{n \tau_{\varepsilon}^{+}} \circ \phi_{X_{\varepsilon}^{+}}^{\varepsilon}(m)\right) \leq 2 C e^{-n \tau \lambda},
$$

where $\tau=\min _{\varepsilon} \tau_{\varepsilon}^{+}$. This shows the uniformity with respect to $\varepsilon$ of the limit point

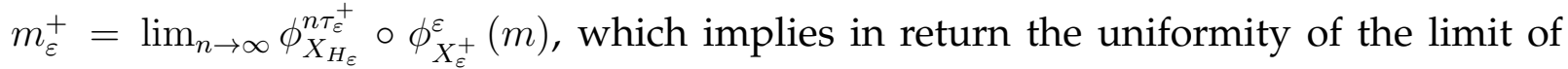
$\int_{0}^{n \tau_{\varepsilon}^{+}} X_{H_{\varepsilon}}(A) \circ \phi_{X_{H_{\varepsilon}}^{t}}^{t} \circ \phi_{X_{\varepsilon}^{+}}^{\varepsilon}(m) d t$. The term $X_{0}^{+}(A)$ is thus given by the expression

$$
X_{0}^{+}(A)=\left.\frac{d}{d \varepsilon} A\left(m_{\varepsilon}^{+}\right)\right|_{\varepsilon=0}-\left.\frac{d}{d \varepsilon} \lim _{n \rightarrow \infty} \int_{0}^{n \tau_{\varepsilon}^{+}} X_{H_{\varepsilon}}(A) \circ \phi_{X_{H_{\varepsilon}}}^{t} \circ \phi_{X_{\varepsilon}^{+}}^{\varepsilon}(m) d t\right|_{\varepsilon=0} .
$$

If we perform a second order Taylor expansion on $H_{\varepsilon}$ with respect to $\varepsilon$, i.e., $H_{\varepsilon}=H_{0}+$ $\varepsilon H_{1}+\varepsilon^{2} K_{\varepsilon}$ with $K_{\varepsilon}$ depending smoothly on $\varepsilon$, then one has

$$
X_{H_{\varepsilon}}(A)=\varepsilon\left\{A, H_{1}+\varepsilon K_{\varepsilon}\right\}
$$

since $\left\{H_{0}, A\right\}=0$. This gives

$$
X_{0}^{+}(A)=\left.\frac{d}{d \varepsilon} A\left(m_{\varepsilon}^{+}\right)\right|_{\varepsilon=0}+\lim _{n \rightarrow \infty} \int_{0}^{n \tau_{0}^{+}}\left\{H_{1}, A\right\} \circ \phi_{X_{H_{0}}}^{t}(m) d t,
$$

for all $m$ on $\mathcal{N}_{\varepsilon, \kappa}^{+}$. A completely similar procedure yields the corresponding expression for the term $X_{0}^{-}(A)$, for all $m$ on $\mathcal{N}_{\varepsilon, \kappa^{\prime}}^{-}$, and we obtain the claimed expression for $\beta\left(X_{A}\right)=$ $X_{0}^{+}(A)-X_{0}^{-}(A)$.

As mentioned in Section 2.2.1, this integral expression is not valid in the whole $\mathcal{N}_{0}$, but rather in $\mathcal{N}_{0, \kappa}^{+} \cap \mathcal{N}_{0, \kappa}^{y-}$ for arbitrarily large $\kappa$. Indeed, one should keep in mind that the convergence of the different limits in this expression becomes worse and worse when one let $m$ get closer to $\gamma_{0}^{+}$or $\gamma_{0}^{-}$. This reflects the so-called "heteroclinic entanglement" phenomenon that occurs near $\gamma_{0}^{+}$and $\gamma_{0}^{-}$.

It might seem to the reader that this integral expression is not very easy to handle, but this is unfortunately the only one available without any further assumptions. In the next two subsections though, we consider special cases for which this expression takes a simpler form. 


\subsubsection{Homoclinic case with a perturbation critical on the orbits}

When the perturbation is critical on the orbits $\gamma_{0}^{+}$and $\gamma_{0}^{-}$, i.e., $d\left(H_{\varepsilon}-H_{0}\right)=0$, then both cycles remain periodic orbits of the perturbed dynamics $H_{\varepsilon}$ for all $\varepsilon$. This means that there is a family of energies $E_{\varepsilon}$ such that the family of orbits $\gamma_{\varepsilon}^{+}$, included in the energy levels $\left\{H_{\varepsilon}=E_{\varepsilon}\right\}$, is actually constant, i.e., $\gamma_{\varepsilon}^{+}=\gamma_{0}$. This would yield a simplification in the formula of Theorem 33 since the term $\left.\frac{d}{d \varepsilon} A\left(m_{\varepsilon}^{+}\right)\right|_{\varepsilon=0}$ would vanish and the integral would converge without prescription on the way to take the limit. Of course, one could do this rather for the unstable orbit $\gamma_{\varepsilon}^{-}$, but unfortunately it is impossible to do this simultaneously for both $\gamma_{\varepsilon}^{+}$and $\gamma_{\varepsilon}^{-}$, except when $H_{\varepsilon}$ takes the same value on $\gamma_{\varepsilon}^{+}$and $\gamma_{\varepsilon}^{-}$. In the heteroclinic situation, this must be an assumption whereas in the homoclinic one this is automatic. Actually, one can obtain this result assuming only that the first order perturbation $H_{1}=\left.\frac{d H_{\varepsilon}}{d \varepsilon}\right|_{\varepsilon=0}$ is critical on $\gamma_{0}^{+}$and $\gamma_{0}^{-}$, as the next theorem shows.

Theorem 34. Let $H_{0} \in C^{\infty}(\mathcal{M})$ be a Hamiltonian either in the heteroclinic or in the homoclinic situation. Let $H_{\varepsilon} \in C^{\infty}(\mathcal{M})$ be a perturbed Hamiltonian such that the first order perturbation $H_{1}$ is critical on both orbits $\gamma_{0}^{+}$and $\gamma_{0}^{-}$. Moreover, in the heteroclinic situation, assume that $H_{1}\left(\gamma_{0}^{+}\right)=H_{1}\left(\gamma_{0}^{-}\right)$. Let $\gamma_{\varepsilon}^{ \pm}$be families of periodic orbits of $H_{\varepsilon}$ included in the energy levels $\left\{H_{\varepsilon}=E_{\varepsilon}\right\}$, with $E_{1}=H_{1}\left(\gamma_{0}^{ \pm}\right)$and let $\beta \in \Omega^{1}\left(\mathcal{N}_{0}\right)$ be the associated Mel'nikov 1-form. Then, for any conserved quantity $A \in C^{\infty}(\mathcal{M})$ and any point $m \in \mathcal{N}_{0} \backslash\left(\gamma_{0}^{+} \cup \gamma_{0}^{-}\right)$, the following formula holds :

$$
\beta\left(X_{A}\right)_{m}=\int_{-\infty}^{+\infty}\left\{H_{1}, A\right\} \circ \phi_{X_{H_{0}}}^{t}(m) d t .
$$

Proof. First of all, $\beta\left(X_{A}\right)_{m}$ is given by the formula of Theorem 33. Since $m_{\varepsilon}^{+} \in \gamma_{\varepsilon}^{+}$one must have $\phi_{X_{H_{\varepsilon}}^{+}}^{\tau^{+}}\left(m_{\varepsilon}^{+}\right)=m_{\varepsilon}^{+}$for all $\varepsilon$, where $\tau_{\varepsilon}^{+}$is the period of $\gamma_{\varepsilon}^{+}$. Let us denote by $Y \in T_{m_{0}^{+}} \mathcal{M}$ the vector tangent to the curve $m_{\varepsilon}^{+}$at $\varepsilon=0$, and let us prove that $Y$ is tangent to $\gamma_{0}^{+}$. For any function $f \in C^{\infty}(\mathcal{M})$ one has $\left.\frac{d}{d \varepsilon} f\left(m_{\varepsilon}^{+}\right)\right|_{\varepsilon=0}=Y(f)$ and the previous equality of curves provides

$$
Y(f)=\left.\frac{d}{d \varepsilon} f \circ \phi_{X_{H_{0}}^{+}}^{\tau^{+}}\left(m_{\varepsilon}^{+}\right)\right|_{\varepsilon=0}+\left.\frac{d}{d \varepsilon} f \circ \phi_{X_{H_{0}}^{+}}^{\tau_{\varepsilon}^{+}}\left(m_{0}^{+}\right)\right|_{\varepsilon=0}+\left.\frac{d}{d \varepsilon} f \circ \phi_{X_{H_{\varepsilon}}^{+}}^{\tau^{+}}\left(m_{0}^{+}\right)\right|_{\varepsilon=0} .
$$

The first term is simply $Y\left(f \circ \phi_{X_{H_{0}}}^{\tau_{0}^{+}}\right)$, i.e., $\left(\left(\phi_{X_{H_{0}}}^{\tau_{0}^{+}}\right)_{*} Y\right) f$. The second one is $\tau_{1} X_{H_{0}}(f) \circ$ $\phi_{X_{H_{0}}^{+}}^{\tau_{0}^{+}}\left(m_{0}^{+}\right)$which is equal to $\tau_{1} X_{H_{0}}(f)_{m_{0}}$. And the third one is a variation of a flow whose expression is

$$
\left.\frac{d}{d \varepsilon} f \circ \phi_{X_{H_{\varepsilon}}}^{\tau_{0}^{+}}\left(m_{0}^{+}\right)\right|_{\varepsilon=0}=\int_{0}^{\tau_{0}^{+}}\left(\left(\phi_{X_{H_{0}}}^{\tau_{0}^{+}}\right)_{*} X_{H_{1}}\right)_{\phi_{X_{H_{0}}}^{\tau_{+}^{+}}\left(m_{0}^{+}\right)} f d t
$$

But this vanishes since by hypothesis $d H_{1}=0$ at each point of $\gamma_{0}^{+}$and thus $X_{H_{1}}=0$ on $\gamma_{0}^{+}$. All together, these terms give the following equation at the point $m_{0}^{+}$

$$
Y=\left(\phi_{X_{H_{0}}}^{\tau_{0}^{+}}\right)_{*} Y+\tau_{1} X_{H_{0}}
$$


Now, remember that $\left(\phi_{X_{H_{0}}}^{\tau_{0}^{+}}\right)_{*}$ at the point $m_{0}^{+}$has the eigenvalue 1 with multiplicity 2, whose eigenspace contains $X_{H_{0}}$. Decompose $Y$ accordingly, i.e., as $Y=Y_{1}+Y_{2}$, with $\left(\phi_{X_{H_{0}}}^{\tau^{+}}\right)_{*} Y_{1}=Y_{1}$ and $Y_{2}$ in the sum of the other eigenspaces. Therefore, the component $Y_{1}$ satisfies $Y_{1}=Y_{1}+\tau_{1} X_{H_{0}}$ which proves that $\tau_{1}=0$ and that $\left(\phi_{X_{H_{0}}}^{\tau_{0}^{+}}\right)_{*} Y=Y$. On the other hand, differentiating the relation $H_{\varepsilon}\left(m_{\varepsilon}^{+}\right)=E_{\varepsilon}$ with respect to $\varepsilon$ yields $Y\left(H_{0}\right)+$ $H_{1}\left(m_{0}^{+}\right)=E_{1}$ and the hypothesis $E_{1}=H_{1}\left(\gamma_{0}^{ \pm}\right)$implies that $Y\left(H_{0}\right)=0$. Together with the fact that $Y$ is an eigenvector of $\left(\phi_{X_{H_{0}}}^{\tau_{0}^{+}}\right)_{*}$ with eigenvalue 1 , this shows that $Y$ is collinear to $X_{H_{0}}$, i.e., tangent to $\gamma_{0}^{+}$. Arguing exactly in the same way, we show that $Y$ is also tangent to the second orbit $\gamma_{0}^{-}$. This shows that, in the formula of Theorem 33 , both terms $\left.\frac{d}{d \varepsilon} A\left(m_{\varepsilon}^{+}\right)\right|_{\varepsilon=0}$ and $\left.\frac{d}{d \varepsilon} A\left(m_{\varepsilon}^{-}\right)\right|_{\varepsilon=0}$ vanish.

On the other hand, the Poisson bracket $\left\{H_{1}, A\right\}$ vanishes on the orbits $\gamma_{0}^{+}$and $\gamma_{0}^{-}$ since $d H_{1}$ does. This implies that the integral $\int_{-T}^{T}\left\{H_{1}, A\right\} \circ \phi_{X_{H_{0}}}^{t}(m) d t$ converges when $T \rightarrow \infty$ and one can replace $\lim _{n \rightarrow \infty} \int_{-n \tau_{0}^{-}}^{n \tau_{0}^{+}}$by $\int_{-\infty}^{+\infty}$.

\subsubsection{The shrewd choice of the conserved quantity}

Suppose now that the perturbation does not have any special properties. We first show that when the conserved quantity $A$ is critical on the periodic orbits, then the expression of Theorem 33 simplifies as in Theorem 34. This result is proved in Theorem 35 and then, we explain how many $A^{\prime}$ s with this property one can have.

Theorem 35. Let $H_{0} \in C^{\infty}(\mathcal{M})$ be a Hamiltonian either in the heteroclinic or in the homoclinic situation. Let $H_{\varepsilon} \in C^{\infty}(\mathcal{M})$ be a perturbed Hamiltonian. Let $\gamma_{\varepsilon}^{ \pm}$be families of periodic orbits of $H_{\varepsilon}$ and let $\beta \in \Omega^{1}\left(\mathcal{N}_{0}\right)$ be the associated Mel'nikov 1-form. Then, for any point $m \in \mathcal{N}_{0} \backslash$ $\left(\gamma_{0}^{+} \cup \gamma_{0}^{-}\right)$and any conserved quantity $A \in C^{\infty}(\mathcal{M})$ which is critical on both orbits $\gamma_{0}^{+}$and $\gamma_{0}^{-}$ , one has the following formula

$$
\beta\left(X_{A}\right)_{m}=\int_{-\infty}^{+\infty}\left\{H_{1}, A\right\} \circ \phi_{X_{H_{0}}}^{t}(m) d t .
$$

Proof. We start from the expression given in Theorem 33 , The vanishing of $d A$ on $\gamma_{0}^{+}$and $\gamma_{0}^{-}$implies that $\left\{H_{1}, A\right\}$ vanishes on $\gamma_{0}^{+}$and $\gamma_{0}^{-}$too. Therefore, the integral $\int_{-T}^{T}\left\{H_{1}, A\right\} \circ$ $\phi_{X_{H_{0}}}^{t}(m) d t$ converges when $T \rightarrow \infty$ and we have $\lim _{n \rightarrow \infty} \int_{-n \tau_{0}^{-}}^{n \tau^{+}}=\int_{-\infty}^{+\infty}$. Moreover we have obviously $\left.\frac{d}{d \varepsilon} A\left(m_{\varepsilon}^{+}\right)\right|_{\varepsilon=0}=0$ and $\left.\frac{d}{d \varepsilon} A\left(m_{\varepsilon}^{-}\right)\right|_{\varepsilon=0}=0$, and this provides the claimed expression.

Let us now address the issue of counting how many such conserved quantities with this property one can have. Remark, that we need only $d-1$ independent $A_{j}$ 's in order to describe completely $\beta$, thru the evaluations $\beta\left(X_{A_{j}}\right)$, since $H_{0}$ itself is a conserved quantity and we know already from Lemma 23 that $\beta\left(X_{H_{0}}\right)=0$. 
Proposition 36. Suppose $H_{0}$ admits a momentum map regular on $\mathcal{N}_{0} \backslash\left(\gamma_{0}^{+} \cup \gamma_{0}^{-}\right)$and define $p$ by

$$
p=\left\{\begin{array}{c}
d-1 \text { in the homoclinic situation } \\
d-2 \text { in the heteroclinic situation. }
\end{array}\right.
$$

Then, there exist $p$ commuting constants of the motion $B_{1}, \ldots, B_{p}$ which are critical on both $\gamma_{0}^{+}$ and $\gamma_{0}^{-}$, and satisfy

$$
d H_{0} \wedge d B_{1} \wedge \ldots \wedge d B_{p} \neq 0 \text { on } \mathcal{N}_{0} \backslash\left(\gamma_{0}^{+} \cup \gamma_{0}^{-}\right) .
$$

Proof. First, let us define the $d$-dimensional vector space $E \subset C^{\infty}(\mathcal{M})$ generated by the components $\left(A_{1}, \ldots, A_{d}\right)$ of the momentum map. Proposition 32 implies that for each $A \in E$ there is a real number $c^{ \pm}(A)$ such that $X_{A}-c^{ \pm}(A) X_{H}$ vanishes on the orbit $\gamma_{0}^{ \pm}$. Any function $A \in E$ is critical on $\gamma_{0}^{ \pm}$precisely when $c^{ \pm}(A)=0$. Actually, the map $A \rightarrow c^{ \pm}(A)$ is linear with respect to $A \in E$. Indeed, one has $X_{A+A^{\prime}}=X_{A}+X_{A^{\prime}}$ which equals to $\left(c^{ \pm}(A)+c^{ \pm}\left(A^{\prime}\right)\right) X_{H}$ on the orbit $\gamma_{0}^{ \pm}$, and similarly, for any constant $\lambda$ one has $X_{\lambda A}=\lambda X_{A}$ which equals to $\lambda c^{ \pm}(A) X_{H}$ on $\gamma_{0}^{ \pm}$. Moreover, this map is non trivial. Indeed, since the $X_{A_{1}}, \ldots, X_{A_{d}}$ form a basis of the tangent $T_{m} \mathcal{N}_{0}$ at each point $m \in \mathcal{N}_{0} \backslash\left(\gamma_{0}^{+} \cup \gamma_{0}^{-}\right)$, the vector field $X_{H}$ restricted to $\mathcal{N}_{0} \backslash\left(\gamma_{0}^{+} \cup \gamma_{0}^{-}\right)$is of the form $X_{H}=\Sigma_{j} a_{j} X_{A_{j}}$, with $a_{j} \in C^{\infty}\left(\mathcal{N}_{0} \backslash\left(\gamma_{0}^{+} \cup \gamma_{0}^{-}\right)\right)$. Now, since the $X_{A_{j}}$ 's commute with each other and with $X_{H}$, this implies that the functions $a_{j}$ are constant on $\mathcal{N}_{0} \backslash\left(\gamma_{0}^{+} \cup \gamma_{0}^{-}\right)$. Therefore, $X_{H}$ coincides with $X_{A}$ on $\mathcal{N}_{0} \backslash\left(\gamma_{0}^{+} \cup \gamma_{0}^{-}\right)$, where $A \in E$ is given by $A=$ $\Sigma_{j} a_{j} A_{j}$. By continuity, they coincide on the whole $\mathcal{N}_{0}$. For this $A$, the map $c^{ \pm}$thus gives 1. Therefore, the set $\left(c^{ \pm}\right)^{-1}(0)$ is a $d-1$ dimensional hyperplane in $E$ composed of first integrals $A$ which are critical on $\gamma_{0}^{ \pm}$. In the heteroclinic case, the hyperplanes $\left(c^{+}\right)^{-1}(0)$ and $\left(c^{-}\right)^{-1}(0)$ do generically not coincide and therefore intersect along a $d-2$ dimensional plane in $E$.

Unfortunately, in the heteroclinic case, one can not avoid that only $d-2$ functions $B_{j}$ are provided by Proposition 36. The systems usually presented in the literature (2dimensional time-periodic) are particular in this regard, because the flow of $H_{0}$ has the same period on $\gamma_{0}^{+}$and $\gamma_{0}^{-}$. In that case, there is indeed $d-1$ functions $B_{j}$, as the next proposition shows.

Proposition 37. Suppose $H_{0}$ admits a momentum map regular on $\mathcal{N}_{0} \backslash\left(\gamma_{0}^{+} \cup \gamma_{0}^{-}\right)$. Assume that the periods of $\gamma_{0}^{+}$and $\gamma_{0}^{-}$are equal, then there exist $d-1$ commuting constants of the motion $B_{1}, \ldots, B_{p}$ which are critical on both $\gamma_{0}^{+}$and $\gamma_{0}^{-}$, and satisfy

$$
d H_{0} \wedge d B_{1} \wedge \ldots \wedge d B_{p} \neq 0 \text { on } \mathcal{N}_{0} \backslash\left(\gamma_{0}^{+} \cup \gamma_{0}^{-}\right) .
$$

Proof. First, let $\tau_{0}^{ \pm}$be the period of $\gamma_{0}^{ \pm}$. We recall that the stable manifold $\mathcal{N}^{+}\left(\gamma_{0}^{+}\right)$of the orbit $\gamma_{0}^{+}$is fibred by the stable manifolds $\mathcal{N}^{+}\left(m^{+}\right)$of all the points $m^{+} \in \gamma_{0}^{+}$, i.e., for each $m \in \mathcal{N}^{+}\left(\gamma_{0}^{+}\right)$, the sequence $\phi_{X_{H_{0}}^{n}}^{n \tau^{+}}(m)$ tends to a point $m^{+} \in \gamma_{0}^{+}$when $n \rightarrow+\infty$. Moreover, the map $m \rightarrow m^{+}$is smooth, i.e., the limit $\pi=\lim _{n} \phi_{X_{H_{0}}}^{n \tau_{0}^{+}}$acts as a projection. Of course, the same holds for the unstable manifold $\mathcal{N}^{-}\left(\gamma_{0}^{-}\right)$of the orbit $\gamma_{0}^{-}$. 
On the other hand, if $A \in C^{\infty}(\mathcal{M})$ is any conserved quantity, then $\left(\phi_{X_{H_{0}}}^{n \tau_{0}^{+}}\right)_{*} X_{A}=X_{A}$ for all $n$. Therefore, $X_{A}$ is tangent to $\mathcal{N}^{+}\left(m^{+}\right)$at a point $m$ iff $X_{A}$ vanishes at $m^{+}$and thus on the whole $\gamma_{0}^{+}$. Now, if the periods $\tau_{0}^{+}$and $\tau_{0}^{-}$are equal, then for each $m^{+} \in \gamma_{0}^{+}$there is a point $m^{-} \in \gamma_{0}^{-}$such that the manifolds $\mathcal{N}^{+}\left(m^{+}\right)$and $\mathcal{N}^{-}\left(m^{-}\right)$coincide. Consequently, $X_{A}$ vanishes at $m^{+}$iff it does at $m^{-}$. Following the proof of Proposition 36, one builds functions $B_{j}$ which are critical on $\gamma_{0}^{+}$and automatically on $\gamma_{0}^{-}$too.

Unfortunately, the systems where the periods are equal are non-generic. We hope that the reader will get convinced by the following example in dimension 4 (but can easily be adapted to higher dimensions).

Example 38. Consider the symplectic manifold $\mathcal{M}=\frac{\mathbb{R}}{\mathbb{Z}} \times \mathbb{R} \times \mathbb{R}^{2}$, with the symplectic form $\omega=d \eta \wedge d t+d \xi \wedge d x$. Let $F(x, \xi)=\xi^{2}+\cos x$. Fix a small $\delta>0$. Let $G(x, \xi)$ a smooth function compactly supported in $\left\{x^{2}+\xi^{2} \leq \delta\right\}$. Assume that $G=c>0$ in the disc $\left\{x^{2}+\xi^{2} \leq \frac{\delta}{2}\right\}$. Now, consider the Hamiltonian $H \in C^{\infty}(\mathcal{M})$ defined by

$$
H(t, \eta, x, \xi)=\eta(1+G(x, \xi))+F(x, \xi) .
$$

First, $H$ is completely integrable since it obviously Poisson-commutes with $\eta$. One can check that for $\eta$ sufficiently small, the transversally hyperbolic periodic orbits of $H$ are $\gamma_{\eta, p}(t)=(t, \eta, p, 0)$, with $p \in 2 \pi \mathbb{Z}$. The picture below represents a Poincaré section $(\eta$ fixed and $t=0$ ) of the flow of $H$.

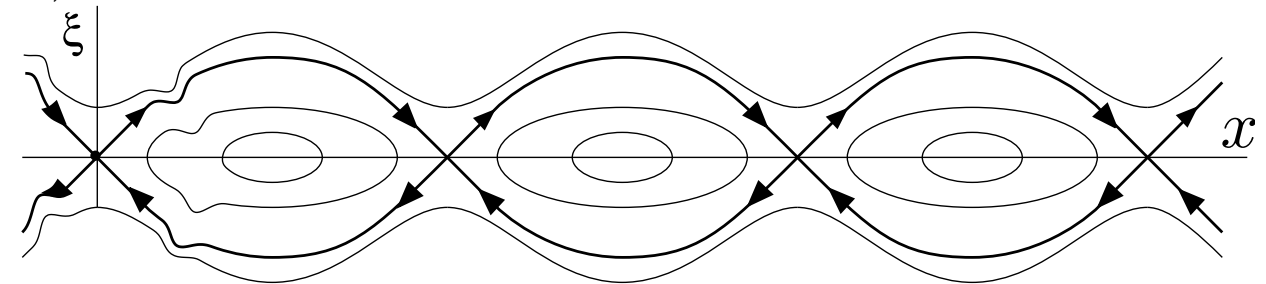

Then, a short calculation shows that all the periodic orbits $\gamma_{\eta, p}$ for $p \neq 0$ have period 1 while $\gamma_{\eta, 0}$ has period $\frac{1}{1+c}$. In fact, this example is very general. One can work on $\mathcal{M}=T^{*} S^{1} \times \mathcal{M}_{0}$, with any symplectic manifold $\mathcal{M}_{0}$. It is enough to choose a function $F \in C^{\infty}\left(\mathcal{M}_{0}\right)$ with hyperbolic critical points linked by heteroclinic manifolds as in the picture above, and a function $G \in C^{\infty}\left(\mathcal{M}_{0}\right)$ compactly supported around one of these critical points as above.

\subsubsection{Remark on Mel'nikov potentials}

We would like to conclude this section with a short remark on Mel'nikov potentials. As mentioned in the introduction, it might happen that the Mel'nikov 1-form $\beta$ admits a nice (convergent) integral expression whereas the Mel'nikov potentials do not (although the potential itself always exists, as shown in Section 2.2.3). Indeed, suppose that we are in the situation of Proposition 37, i.e., the periods on $\gamma_{0}^{+}$and $\gamma_{0}^{-}$are equal. In that case, one has $d$ commuting constants of the motion $A_{1}, \ldots, A_{d}$ for which $\beta\left(X_{A_{j}}\right)$ admits the integral expression given in Theorem 35, namely $A_{1}, \ldots, A_{d-1}$ are 
those given by Proposition 37 and $A_{d}=H_{0}$. Moreover, the associated Hamiltonian vector fields $X_{j}:=X_{A_{j}}$ provide a global frame on $\mathcal{N}_{0} \backslash\left(\gamma_{0}^{+} \cup \gamma_{0}^{-}\right)$. Therefore, provided a origin point $m_{0} \in \mathcal{N}_{0} \backslash\left(\gamma_{0}^{+} \cup \gamma_{0}^{-}\right)$is fixed, one can parameterise ${ }^{3} \mathcal{N}_{0} \backslash\left(\gamma_{0}^{+} \cup \gamma_{0}^{-}\right)$by $\left(t_{1}, \ldots, t_{d}\right) \in \mathbb{R}^{d} \rightarrow m=\phi_{X_{1}}^{t_{1}} \circ \ldots \circ \phi_{X_{d}}^{t_{d}}\left(m_{0}\right)$. A Mel'nikov potential $L$ is well-defined up to a constant which can be fixed by setting $L\left(m_{0}\right)=0$. Then, one has

$$
L(m)=\int_{0}^{1} \frac{d}{d s} L \circ \phi_{\sum_{j} t_{j} X_{j}}\left(m_{0}\right) d s=\int_{0}^{1} \sum_{j} t_{j} \beta\left(X_{j}\right) \phi_{\sum_{j} t_{j} X_{j}}^{s}\left(m_{0}\right) d s
$$

since the flows of the $X_{j}$ 's commute with each other. Inserting the integral expression of $\beta$ and exchanging the order of the sum $\Sigma_{j}$ and the integral $\int d t$, one obtains

$$
L(m)=\int_{0}^{1}\left(\int_{-\infty}^{+\infty}\left\{H_{1}, \sum_{j} t_{j} A_{j}\right\} \circ \phi_{X_{H_{0}}}^{t} \circ \phi_{\sum_{j} t_{j} X_{j}}^{s}\left(m_{0}\right) d t\right) d s .
$$

Now, since $X_{H_{0}}$ is a symplectic vector field commuting with the $X_{j}{ }^{\prime}$ s, one has simply

$$
\left\{H_{1}, \sum_{j} t_{j} A_{j}\right\} \circ \phi_{X_{H_{0}}}^{t}=\left\{H_{1} \circ \phi_{X_{H_{0}}}^{t}, \sum_{j} t_{j} A_{j}\right\} \text {. }
$$

Finally, if it was possible to exchange the order of the two integrals $\int d s$ and $\int d t$, then we would get

$$
L(m)=\int_{-\infty}^{+\infty}\left(-\int_{0}^{1} \frac{d}{d s} H_{1} \circ \phi_{X_{H_{0}}}^{t} \circ \phi_{\sum_{j} t_{j} X_{j}}^{s}\left(m_{0}\right) d s\right) d t .
$$

The integration over the $s$ variable would give

$$
L(m)=\int_{-\infty}^{+\infty}\left(H_{1} \circ \phi_{X_{H_{0}}}^{t}\left(m_{0}\right)-H_{1} \circ \phi_{X_{H_{0}}}^{t}(m)\right) d t .
$$

Unfortunately, this integral is not convergent unless we assume that $H_{1}$ is constant on the orbits $\gamma_{0}^{ \pm}$(in particular this is the case when $H_{1}$ is critical on $\gamma_{0}^{ \pm}$).

\subsection{Recovering the Mel'nikov function}

The Mel'nikov "function" was historically introduced for studying periodically forced 2-dimensional Hamiltonian systems. We present here the class of periodically forced system, as a special example of the general framework we have been developing throughout this article.

Let $(\mathcal{M}, \omega)$ be a $2 d$-dimensional symplectic manifold and $H_{0} \in C^{\infty}(\mathcal{M})$ a Hamiltonian admitting two fixed points $m_{0}^{+}$and $m_{0}^{-}$, which are hyperbolic in the sense that the

\footnotetext{
${ }^{3}$ This parameterisation is not injective, but it is surjective.
} 
linear maps which sends $Y \in T_{m_{0}^{ \pm}} \mathcal{M}$ to $\left[\tilde{Y}, X_{H_{0}}\right]_{m_{0}^{{ }^{\prime}}}$, for any extension $\tilde{Y} \in \Gamma(T \mathcal{M})$, has no eigenvalue on the imaginary axis. This implies the existence of stable and unstable manifolds for both points, and we suppose that the stable manifold $\mathcal{N}_{0}^{+}$of $m_{0}^{+}$coincides with the unstable manifold $\mathcal{N}_{0}^{-}$of $m_{0}^{-}$. We suppose moreover, that $H_{0}$ is completely integrable, i.e., there is a momentum map $\left(A_{1}, \ldots, A_{d}\right)$. This hypothesis is automatically true in the 2-dimensional case usually considered. Then, we perturb the Hamiltonian into a 1-periodic time-dependent Hamiltonian $H_{\varepsilon}(t)$. For studying such systems, it is very convenient to consider the "extended system" on the $(2 d+2)$-dimensional manifold $\tilde{\mathcal{M}}=\mathcal{M} \times T^{*} S^{1}$, where the $S^{1}$ factor corresponds to the $t$ variable. This manifold is equipped with the symplectic form $\pi^{*} \omega+d \eta \wedge d t$, where $\eta$ is the moment variable associated with $t$ and $\pi$ is the projection $\tilde{\mathcal{M}} \rightarrow \mathcal{M}$. Let us denote the Poisson brackets on $\tilde{\mathcal{M}}$ (resp. $\mathcal{M})$ by $\{,\}^{\sim}$ (resp. $\left.\{\},\right)$.

The perturbed Hamiltonian $H_{\varepsilon}$ can be viewed as a function on $\tilde{\mathcal{M}}$ independent on $\eta$. Then, we define the extended Hamiltonian $\tilde{H}_{\varepsilon}=H_{\varepsilon} \circ \pi+\eta$ and it is easy to check that the dynamics of $H_{\varepsilon}$ is given by the projection on $\mathcal{M}$ of the dynamics of $\tilde{H}_{\varepsilon}$ on $\tilde{\mathcal{M}}$.

Since the points $m_{0}^{ \pm}$are fixed for $H_{0}$, they give rise to periodic orbits $t \rightarrow\left(m_{0}^{ \pm},(\eta, t)\right)$ for $\tilde{H}_{\varepsilon}$, denoted by $\gamma_{0}^{ \pm}$, and the hyperbolicity of $m_{0}^{ \pm}$implies the one of $\gamma_{0}^{ \pm}$. The stable manifold of $\gamma_{0}^{+}$, denoted by $\tilde{\mathcal{N}}_{0}^{+}$, is nothing but the union over all $s \in S^{1}$ of $\phi_{\partial_{t}}^{s}\left(\mathcal{N}_{0}^{+}\right)$, and coincides with $\tilde{\mathcal{N}}_{0}^{-}$, the unstable manifold of $\gamma_{0}^{-}$. The pull-back to $\tilde{\mathcal{M}}$ of the momentum map provides $d$ functions $\tilde{A}_{j}=A_{j} \circ \pi$ which are invariant by the flow of $\tilde{H}_{0}$, since $\left\{\tilde{H}_{0}, \tilde{A}_{j}\right\}_{(m,(\eta, t))}^{\sim}=\left\{H_{0}, A_{j}\right\}_{m}=0$. Moreover, they are critical on $\gamma_{0}^{ \pm}$since the $A_{j}$ 's are critical on $m_{0}^{ \pm}$, because of the hyperbolicity of $m_{0}^{ \pm}$. This means that we can apply Theorem 35] which says that the Mel'nikov 1-form $\beta$ evaluated on the $X_{\tilde{A}_{j}}$ 's gives

$$
\beta\left(X_{\tilde{A}_{j}}\right)_{(m,(\eta, t))}=\int_{-\infty}^{+\infty}\left\{H_{1}, \tilde{A}_{j}\right\}^{\sim} \circ \phi_{X_{\tilde{H}_{0}}}^{s}(m,(\eta, t)) d s .
$$

Now, the flows of $\tilde{H}_{0}$ and $H_{0}$ are simply related by

$$
\phi_{X_{\tilde{H}_{0}}}^{s}(m,(\eta, t))=\left(\phi_{X_{H_{0}}}^{s}(m),(\eta, t+s)\right) .
$$

Moreover, since the $\tilde{A}_{j}$ 's are pullbacks, then $\left\{H_{1}, \tilde{A}_{j}\right\}_{(m,(\eta, t))}^{\sim}$ is simply equal to $\left\{H_{1}(t), A_{j}\right\}_{m}$. Therefore, the evaluation of $\beta$ becomes

$$
\beta\left(X_{\tilde{A}_{j}}\right)_{(m,(\eta, t))}=\int_{-\infty}^{+\infty}\left\{H_{1}(t+s), A_{j}\right\} \circ \phi_{X_{H_{0}}}^{s}(m) d s,
$$

which is the usual form of the so-called "Mel'nikov functions" $M_{j}(t)$, for a fixed point $m$. 


\section{References}

[1] R. Abraham and J. Marsden. Foundations of Mechanics. Benjamin/Cummings Publishing Co. Inc. Advanced Book Program, seconde edition, 1978.

[2] V. I. Arnol'd. Instability of dynamical systems with many degrees of freedom. Dokl. Akad. Nauk SSSR, 156:9-12, 1964.

[3] L. Chierchia and G. Gallavotti. Drift and diffusion in phase space. Ann. Inst. H. Poincaré Phys. Théor., 60(1):144, 1994.

[4] Y. Colin de Verdière and S. Vũ Ngọc. Singular Bohr-Sommerfeld rules for 2D integrable systems. Ann. Sci. École Norm. Sup. (4), 36(1):1-55, 2003.

[5] A. Delshams and P. Gutiérrez. Splitting potential and the Poincaré-Melnikov method for whiskered tori in Hamiltonian systems. J. Nonlinear Sci., 10(4):433-476, 2000 .

[6] A. Delshams and P. Gutiérrez. Homoclinic orbits to invariant tori in Hamiltonian systems. In Multiple-time-scale dynamical systems (Minneapolis, MN, 1997), volume 122 of IMA Vol. Math. Appl., pages 1-27. Springer, New York, 2001.

[7] L. H. Eliasson. Biasymptotic solutions of perturbed integrable Hamiltonian systems. Bol. Soc. Brasil. Mat. (N.S.), 25(1):57-76, 1994.

[8] N. Fenichel. Persistence and smoothness of invariant manifolds for flows. Indiana Univ. Math. J., 21:193-226, 1971/1972.

[9] P. J. Holmes and J. E. Marsden. Mel'nikov's method and Arnol'd diffusion for perturbations of integrable Hamiltonian systems. J. Math. Phys., 23(4):669-675, 1982.

[10] P. Lochak, J.-P. Marco, and D. Sauzin. On the splitting of invariant manifolds in multidimensional near-integrable Hamiltonian systems. Mem. Amer. Math. Soc., 163(775), 2003.

[11] V. K. Mel'nikov. On the stability of a center for time-periodic perturbations. Trudy Moskov. Mat. Obšč., 12:3-52, 1963.

[12] Zung Nguyen Tien. Symplectic topology of integrable Hamiltonian systems. I. Arnold-Liouville with singularities. Compositio Math., 101(2):179-215, 1996.

[13] A. Weinstein. Symplectic manifolds and their lagrangian submanifolds. Adv. in Math., 6:329-346, 1971.

[14] S. Wiggins. Global bifurcations and chaos, volume 73 of Applied Mathematical Sciences. Springer-Verlag, 1988. Analytical methods. 